\title{
Deciphering the olfactory repertoire of the tiger mosquito Aedes albopictus
}

\author{
Fabrizio Lombardo ${ }^{1 *} \mathbb{D}$, Marco Salvemini ${ }^{2}$, Carmine Fiorillo', Tony Nolan ${ }^{3}$, Laurence J. Zwiebel ${ }^{4}$, José M. Ribeiro ${ }^{5}$ \\ and Bruno Arcà
}

\begin{abstract}
Background: The Asian tiger mosquito Aedes albopictus is a highly invasive species and competent vector of several arboviruses (e.g. dengue, chikungunya, Zika) and parasites (e.g. dirofilaria) of public health importance. Compared to other mosquito species, Ae. albopictus females exhibit a generalist host seeking as well as a very aggressive biting behaviour that are responsible for its high degree of nuisance. Several complex mosquito behaviours such as host seeking, feeding, mating or oviposition rely on olfactory stimuli that target a range of sensory neurons localized mainly on specialized head appendages such as antennae, maxillary palps and the mouthparts.

Results: With the aim to describe the Ae. albopictus olfactory repertoire we have used RNA-seq to reveal the transcriptome profiles of female antennae and maxillary palps. Male heads and whole female bodies were employed as reference for differential expression analysis. The relative transcript abundance within each tissue (TPM, transcripts per kilobase per million) and the pairwise differential abundance in the different tissues (fold change values and false discovery rates) were evaluated. Contigs upregulated in the antennae (620) and maxillary palps (268) were identified and relative GO and PFAM enrichment profiles analysed. Chemosensory genes were described: overall, 77 odorant binding proteins (OBP), 82 odorant receptors (OR), 60 ionotropic receptors (IR) and 30 gustatory receptors (GR) were identified by comparative genomics and transcriptomics. In addition, orthologs of genes expressed in the female/male maxillary palps and/or antennae and involved in thermosensation (e.g. pyrexia and arrestin1), mechanosensation (e.g. piezo and painless) and neuromodulation were classified.
\end{abstract}

Conclusions: We provide here the first detailed transcriptome of the main Ae. albopictus sensory appendages, i.e. antennae and maxillary palps. A deeper knowledge of the olfactory repertoire of the tiger mosquito will help to better understand its biology and may pave the way to design new attractants/repellents.

Keywords: Mosquito, Aedes albopictus, Olfaction, Antennae, Maxillary palps, mRNA-sequencing, Chemosensory genes

\section{Background}

The Asian tiger mosquito Aedes albopictus is an aggressive daytime-biting vector of several arboviruses pathogenic to humans (e.g. dengue, chikungunya, Zika). Ae. albopictus has been described as one of the 100 worst invasive species in the world (Global Invasive Species Database, http://www.issg.org/database/). Its impact on human health relies indeed on its rapid and aggressive worldwide spread from its native home range (South-East Asia), along with its ecological adaptability in different

\footnotetext{
* Correspondence: fabrizio.lombardo@uniroma1.it

${ }^{1}$ Department of Public Health and Infectious Diseases, Division of

Parasitology, Sapienza University of Rome, Rome, Italy

Full list of author information is available at the end of the article
}

traits, including most importantly feeding behaviours, diapause, and vector competence [1-5].

Biological signals captured from the surrounding environment and sensed through olfaction and other chemosensory modalities play a central role in the modulation of mosquito behaviours such as hostseeking, feeding, mating, oviposition and reception of repellents [6]. Olfactory responses are initiated by activation of olfactory sensory neurons (OSNs) localized mainly on antennae, maxillary palps, mouthparts (consisting of the proboscis and labellum) and tarsi [6]. These sensory appendages may perceive extremely diverse extrinsic stimuli, such as volatile and non-volatile odours or pheromones, temperature, humidity, mild or noxious touch, gravity, etc., to activate a complex mix of 
mosquito perception pathways [7-9]. The sensing of chemical stimuli, i.e. chemosensation, relies on chemosensory neurons that are selectively activated by various volatile compounds, such as odorant molecules and pheromones [10]. The molecular components underlying peripheral olfactory signalling encompass a range of intracellular and extracellular contexts. The complexity of olfactory factors together with differential expression and/ or abundance directly contributes to the modulation of specific behaviours across mosquito species. From a receptor-centric perspective, chemosensation in insects is largely mediated by diverse members of three gene families expressed primarily in OSNs that reside within specialized sensilla that populate olfactory appendages: odorant receptors (ORs), gustatory receptors (GRs) and ionotropic receptors (IRs) (reviewed in $[6,11]$ ). Odorants and chemical compounds cross the cuticle through sensillar pores to reach the aqueous sensillar lymph, and are then postulated to be recognized and carried to their cognate specific receptors on OSN dendritic membranes by members of a diverse family of extracellular odorant binding proteins (OBPs) and pheromone binding proteins (PBPs) that are secreted by a network of accessory cells localized at the base of insect sensilla (reviewed in [12]). There are several distinct types of chemosensory sensilla which house the OSNs along other sensory neurons and their associated accessory cells that populate mosquito sensory appendages [13].

From a cellular and molecular perspective, several studies concerning the activation of olfactory transduction pathways in insects suggest that ORs function as heteromeric complexes that form ligand gated ion channels in association with the ubiquitous co-receptor ORco $[14,15]$, that may also in some circumstances utilize Gprotein coupled second messenger pathways to confer OSN odour sensitivity [16]. Activation of OSNs by odorants triggers complex behavioural responses [17], and several evidences highlight the crucial role of ORs in accomplishing this process in mosquitoes [18, 19]. Membrane-bound insect GRs are phylogenetically related to ORs and are generally expressed in gustatory receptor neurons (GRNs) found in chemosensory sensilla distributed on the mouthparts, wing margins, genitalia, and tarsal segments of the legs [20-23]. In the malaria mosquito Anopheles gambiae a suite of three highly conserved GRs is expressed in a unique array of non-OR containing maxillary palp OSNs that respond to volatile $\mathrm{CO}_{2}$ [24]. Similarly, in the dengue and yellow fever mosquito Aedes aegypti a crucial role in host preference/ seeking was shown for two of the three GRs as component of the $\mathrm{CO}_{2}$ receptor [25, 26]. Insect IRs are not related to ORs or GRs, but instead represent an ancient family derived from ionotropic glutamate receptors (iGluRs), a highly conserved family of ligand-gated ion channels involved in neurotransmission as well as signalling mechanisms in response to external chemical stimuli in both eukaryotes and prokaryotes [27, 28]. Mosquito IRs are not as well characterized functionally as ORs, although recent studies in An. gambiae [29] are consistent with work from Drosophila melanogaster showing that IRs detect volatile compounds such as acids, ammonia, or amines [30]. Behavioural studies in Ae. aegypti showed that this mosquito uses polyamines both to find feeding sources and especially to locate egglaying sites [31]: this attraction could be mediated by IRs, as demonstrated in the fruit fly [31]. In addition to the principal chemosensory receptor gene families, OSN membranes are also populated with several important families of olfactory genes. The sensory neuron membrane proteins (SNMPs), which belong to the scavenger receptor type B gene family (SCRB/CD36) [32, 33], are receptors involved in cell-cell communications, ligand (fatty acids) internalization [34] and pheromone detection as shown in D. melanogaster [35, 36]. Insect SNMPs are well-conserved and are typically expressed in neurons or support cells associated with sensilla [34].

Beyond those membrane components, a range of secreted proteins makes essential extracellular contributions to olfactory signalling pathways. Of these, OBPs are highly expressed water-soluble components of sensillary lymph that are hypothesized to bind and solubilize odorant compounds from the external environment and to transport them to their respective olfactory receptors triggering olfactory transduction pathways [12]. Insect OBPs are $10-30 \mathrm{kDa}$ globular proteins characterized by six $\alpha$-helical domains comprising of six highly conserved cysteines with specific disulphide connectivity. In mosquitoes three sub-families of OBPs have been characterized so far: (i) Classic OBPs, carrying the six conserved cysteines typical of the OBP family; (ii) PlusC OBPs, with the same conserved cysteines and disulphide connectivity but also containing six additional cysteines; (iii) Atypical OBPs, which are among the longest known OBPs including two domains that are homologous to the Classic OBP domain and are hence considered as "dimer OBPs" [37, 38]. Moreover, OBPs lacking C2 and C5 cysteines are commonly widespread among studied organisms and are named MinusC [38]. Structural studies in mosquito described the adaptability of OBP binding site to accommodate several ligands [39, 40]. It was also demonstrated that some OBP might show a binding preference for certain odour molecules. For instance, the presence of OBP1 in An. gambiae mediates the binding with the ligand indole [41], a known oviposition attractant for the southern house mosquito Culex quinquefasciatus [42]. OBPs therefore likely represent a critical initial interface between the environment and the mosquito: the variety of OBPs and chemosensory receptors 
each mosquito possesses and expresses at any one time is likely to influence specific behavioural features (i.e., food, host and oviposition site preference and seeking) as reflected in their capacity to sense diverse sets of attractants and repellents. In addition to OBPs, there are several other families of secreted proteins involved in peripheral chemosensation. Among these, members of CheA and CheB gene families, which encode small soluble proteins [43], were found expressed in sex-specific patterns and involved in the detection of cuticular hydrocarbons required for the courtship in the fruit fly [44]. CheB protein may interact with degenerin/epithelial $\mathrm{Na}^{+}$channels (pickpocket, ppk) to detect these contact pheromones [43]. The molecular mechanism of CheA and CheB function remains unknown, but may involve interaction with other membrane bound receptors or ppk channels [43]. Another class of secreted extracellular proteins playing crucial roles in olfaction are the odorant/pheromone-degrading enzymes (ODEs/PDEs), which are involved in the degradation of odorants thus clearing sensillar lymph [45].

Sensory appendages such as tarsi and head appendages (labella, proboscis, maxillary palps and antennae) are not only involved in chemosensation but also play essential roles in mediating mosquito sensitivity to various additional stimuli that include temperature, dangerous and mild touch, humidity, gravity and other sensory modalities [6]. In this respect there are several families of membranebound molecules playing relevant roles. The pickpocket (ppk) and the transient receptor potential channels (trp) gene families have potential roles in insect taste, thermoand mechano-reception $[6,46]$. Indeed, mosquitoes not only must avoid extreme cold and heat but also accurately sense temperature as host-seeking females are known to be attracted to a narrow, specific temperature range associated with vertebrate hosts [6]. In D. melanogaster, GR28 has been involved in the detection of rapid temperature changes in adult flies [47]; in mosquitoes, TrpA1, Painless, Pyrexia and $P l c B$ play roles in the detection of harmful heat thresholds and functional studies have directly implicated mosquito TRPA1 in thermo-sensitivity in both $A n$. gambiae [48] and Ae aegypti [49]. In addition, Painless, Pyrexia and $P l c B$ appear also to be involved in light detection. Mechanosensation is the entire repertoire of actions and reactions related to the detection of sound, gravity and mild/noxious touch [46]. Previous studies in the fruit fly identified genes involved in some aspects of mechanosensation: for example the proteins painless, piezo and pickpocket (Ppk1) are mainly involved in perception of noxious touch [50-52], whereas nompC (no mechanoreceptor potential C), Ppk2, chloride channel-b, narrow abdomen, mrityu, the ionotropic receptors Nmdar1 and Nmdar2 are instead required for mild touch detection $[53,54]$.
The worldwide spread of the tiger mosquito Ae. albopictus, its competence in the transmission of several pathogens and the recent involvement in arboviral outbreaks [55-60], point out the need to acquire a deeper knowledge on crucial aspects of its life cycle that may help developing novel and more effective strategies for its control [9]. Here we employ an RNA-seq based approach to generate a comprehensive transcriptome of Ae. albopictus female antennae and maxillary palps. This facilitates the characterization of the main Ae. albopictus gene families involved in the perception of olfactory stimuli as well as of several additional transcripts likely implicated in sensing taste, temperature, humidity, touch, injury and gravity. We believe that the assembly of this olfactory repertoire will be useful for improving the annotation of the Ae. albopictus genome [61, 62]. Moreover, the comparison to other mosquito species may help understanding some particular behavioural and ecological features of the tiger mosquito (niche adaptation, invasiveness, feeding habits) that in the long run it may contribute to the development of novel control strategies.

\section{Methods}

\section{Insects and tissue dissections}

The Aedes albopictus strain used in this study was originally collected in Rome in 2012 and reared in the insectary for several generations (eggs kindly provided by Roberto Romi and Marco Di Luca, Istituto Superiore di Sanità, Rome, Italy). Mosquitoes were reared under standard laboratory conditions $\left(25 \pm 1{ }^{\circ} \mathrm{C}\right.$, relative humidity $60 \pm 10 \%$, light:dark photoperiod $14: 10 \mathrm{~h}$ ) in the insectary of the Department of Public Health and Infectious Diseases at Sapienza University, Rome. Adult females (2-6 days post-emergence, dpe) maintained on a $10 \%$ sucrose diet were used in this study. The age range was selected in the attempt to reveal transcripts encoding most of the olfactory factors involved in the preference, selection and location of blood-meal hosts in female mosquitoes that play critical roles in significantly establishing their vectorial capacity. Indeed, it is known that mosquitoes do not seek blood source in the first 24-48 h post-emergence [63], and temporal expression analyses showed that the largest transcriptional increase of olfactory genes occurs up to day 4 dpe in An. gambiae [64] and up to day 6 in Ae. aegypti, followed by a plateau in 10 dpe mosquitoes [65]. At 2 to $4 \mathrm{~h}$ after the gradual onset of light in rearing chambers ("insectary sunrise") which corresponds to Zeitgeber time (ZT) 2 to ZT4, mosquitoes were anaesthetized using ice (2-3 min), and female antennae, female palps, male heads (with all the appendages attached) were hand-dissected, frozen in liquid nitrogen, and stored at $-80{ }^{\circ} \mathrm{C}$ until needed. Whole females were directly frozen and stored as above. 
Two independent collections of 500 female antennae, 500 female palps, 10 adult females and 15 male heads were used for RNA extractions to obtain two independent biological replicates.

\section{RNA extraction, library preparation and sequencing}

RNA was extracted using Trizol $^{\circ}$ Reagent (Life Technologies) according to the manufacturer's protocol. Dissected tissues and entire females were initially crushed with pestles in Trizol reagent before proceeding with the extraction protocol. RNA concentration and quality were evaluated using standard procedures: Take3 Module (BioTek SynergyHT) reading and gel electrophoresis. RNA samples were treated with DNAseI (Ambion). Amounts of total RNA used to synthesize mRNA TruSeq libraries were the following: 500 Antennae (A): $1.4 \mu \mathrm{g} ; 500$ Antennae (B): $1.9 \mu \mathrm{g} ; 500$ Palps (A): $500 \mathrm{ng}$; 500 Palps (B): 400 ng 10 Female Body (A): $4.5 \mu$ g; 10 Female Body (B): $4.2 \mu \mathrm{g} ; 15$ Male Heads (A): $3.3 \mu \mathrm{g} ; 15$ Male Heads (B): $3 \mu \mathrm{g}$. cDNA library preparation, including fragmentation and barcoding (ligation of specific adapters), was performed following the Illumina TruSeq RNA Library v2 protocol (Illumina, San Diego, CA, USA). After quality evaluation with an Agilent 2010 Bioanalyzer, as recommended for Illumina sequencing, the libraries were diluted with an elution buffer and loaded on an Illumina HiSeq2000 for sequencing (paired-end, PE, $2 \times 100 \mathrm{bp}$ ), and each cDNA library was sequenced.

\section{Assembly, sequence annotation and expression profiling} Clean reads were generated from raw reads by removing adaptor sequences, ambiguous reads, and low-quality reads with a qual value cutoff of 15 . De novo assembly of clean data was accomplished by $\mathrm{ABySS}^{\circ}$ and SOAPdenovo-trans ${ }^{\oplus}$ software, using several k-mers (every 10th from 25 to 95). Quality control between RNA-seq replicates was performed using the PtR Trinity perl script (release 2014-07-17) prior to merging of the duplicate (A/B) assemblies. Merged assembly (BLAST and cap3 assembler) was used to extract putative protein coding sequences (CDS). When the Ae. albopictus genome was made available [61,62], we have compared (by BLAST) the VectorBase AaloF1 gene annotation [62] with the olfactory transcriptome predictions to assign VectorBase ID and reduce redundancy (annotation Software @CBS-DTU). Coding sequences (CDS) were extracted based on the existence of a signal peptide in the longer open reading frame (ORF) and by similarities to other proteins found in the Refseq invertebrate database from the National Center for Biotechnology Information (NCBI), proteins from Diptera deposited at NCBI's Genbank and from SwissProt. To identify gene categories and PFAM terms enriched in the pairwise comparisons between the four tissues a GO/PFAM term enrichment analysis was performed using the Annocript software [66] and the Fisher Exact Test (adjusted $p$-value <0.01) in $\mathrm{R}$ package [67]. Clean reads were mapped back onto the assembled transcriptome using RSEM software and read count for each gene was obtained from the mapping results. Expression levels were assessed in terms of TPM values (transcripts per kilobase per million reads), which were calculated based on the number of mapped transcript fragments corrected for transcript length and sequencing depth. Differential expression analysis of two samples was performed using the edgeR $\mathrm{R}$ package [68]. $P$ value was adjusted using FDR (False Discovery Rate). Then, paired comparisons were conducted in the following manner: female antennae vs. female body, female palps vs. female body and male heads vs. female body. EdgeR was run only for transcripts having at least one library with RPKM equal or larger than one, and only contigs with $2<\operatorname{logFC}<-2$ were considered. Pairwise comparisons using edgeR were done using the generalized linear model (GLM) likelihood ratio test.

\section{Identification of chemosensory genes, sequence alignment and phylogenetic analysis}

As mentioned in the paragraph above, each contig was analysed by BLAST analysis interrogating several databases. Contigs showing significant matches with proteins involved in chemosensation in different insects (the mosquitoes An. gambiae, C. quinquefasciatus and Ae. aegypti and other insects such as the fruit fly $D$. melanogaster) were identified. Moreover, a reciprocal BLAST analysis was carried out using FASTA sequences of $A e$. aegypti OR [69-71], GR [22, 69, 71], IR [27, 69, 71], OBP $[38,69,71]$ and of genes involved in more general sensorial functions [71]. True orthologs were therefore identified by reciprocal best-hit BLASTP analysis screening the Ae. albopictus olfactory transcriptome. These lists were then manually refined by TBLASTN searches taking also into account other relevant biological features such as high percentages of identity over shorter regions or possible gene duplication events. Deduced protein sequences were aligned using Clustal Omega on line tool at EMBL-EBI (http://www.ebi.ac.uk/Tools/msa/ clustalo/). Alignments reported in this manuscript should be considered as provisional and susceptible of improvement, especially considering the limited overall reliability of the present genome annotation. Nevertheless, they provide clear clues and justification for the classification in the different families and subfamilies. Phylograms were obtained by using MEGA 5.0 software and Maximum likelihood protocol. Bootstrapping was performed by the re-sampling amino acid positions of 1000 replicates (Additional file 1). 


\section{Quantitative real-time PCR}

To verify transcript abundance patterns from RNA-seq analysis, RT-qPCR reactions were performed on eleven selected genes. Two novel batches of both antennae and maxillary palps were dissected from Ae. albopictus females 2-5 dpe (days post emergence); two batches of whole female mosquitoes were also collected. Total RNA was extracted using $\mathrm{Trizol}^{\circ}$ reagent (Invitrogen) following manufacturer's instruction and finally resuspended in DNAse-RNAse-free ultrapure $\mathrm{ddH}_{2} \mathrm{O}$. Quantity and quality of RNA was evaluated by spectrophotometric measurement (using the Take3 module of plate reader BioTek SynergyHT and GEN5 ${ }^{\text {max }}$ software) and agarose gel electrophoresis, respectively. DNAseI (using Ambion DNA-free kit $^{\circ}$, per manufacturer's instruction) treatment was followed by standard PCR (without Reverse Transcription) to verify efficacy of treatment. DNAseI-treated total RNA ( $\sim 1 \mu \mathrm{g}$ for dissected tissues and $5 \mu \mathrm{g}$ for whole females) was used as substrate to generate First-Strand cDNA using SuperScript II RT (Invitrogen) and OligodT (Invitrogen). The synthesized cDNA samples were diluted to $5 \mathrm{ng} / \mu \mathrm{l}$ : in each qPCR reaction $2 \mu \mathrm{l}$ of cDNA were used. For each gene, a standard curve was included in each plate, together with the endogenous reference gene (ribosomal protein S5, RpS5, AALF013336, [72, 73]). cDNA templates for the standard curve were obtained from whole female RNA samples, a dilution series starting from $100 \mathrm{ng} /$ reaction was followed by 1:5 dilutions. cDNA templates were mixed with $2 \times$ PowerUp $^{\text {Tw }}$ SYBR $^{\text {ma }}$ Green Master Mix (Applied Biosystem) and specific primers as listed in Additional file 2: Table S6. Each reaction included an initial holding stage of $2 \mathrm{~min}$ at $50{ }^{\circ} \mathrm{C}$ and of $2 \mathrm{~min}$ at $95{ }^{\circ} \mathrm{C}$, followed by 40 cycles of PCR $\left(95{ }^{\circ} \mathrm{C}, 15 \mathrm{~s}\right.$; $60{ }^{\circ} \mathrm{C}, 1 \mathrm{~min}$ ); a final stage to obtain melting curves was also included in each plate, with detection steps every $0.3{ }^{\circ} \mathrm{C}$. In relative quantification, the relative amount of each gene in each tissue is determined by the ratio between the amounts of target gene and endogenous reference gene calculated using their $\mathrm{Ct}$ values and the corresponding standard curves. This ratio is then compared between different samples, choosing a sample as calibrator. The relative transcription levels normalized by reference gene were compared with expression levels of RNA-seq. We initially evaluated the correlation between duplicates in both RNA-seq and qPCR datasets. To obtain values suitable for statistical comparisons, we calculated (for each gene) a fold-change (FC) value as the ratio of abundance over the group median. Duplicates were then averaged to obtain a single FC value for each gene. These values were used to evaluate the correlation between RNA-seq and qPCR methods, applying statistical evaluation throughout Spearman and Pearson tests. For both techniques, statistical evaluation (Spearman and Pearson tests) revealed also a significant linear correlation between duplicates (Additional file 3: Figure S11 A-D).

\section{Results and discussion}

Transcriptome sequencing, de novo assembly and genome release

Duplicate transcriptomes of olfactory appendages of the tiger mosquito Ae. albopictus were obtained for each of the four samples and general assembly statistics are summarized in Table 1 and Fig. 1. A comprehensive transcriptome was produced following de novo assembly procedures. Extracted CDS were compared by BLAST analysis to predicted peptides from the recently released Ae. albopictus genome [AaloF1 database, Foshan strain, [62]]. We found 6069 sequences that had at least 95\% amino acid identity and at least $95 \%$ coverage to the Foshan predictions. Of these, 262 were at least $5 \%$ longer than the predictions. 20,085 contigs had less than 95\% identity to the genomic predictions, and included 972 transcripts that produced $>75 \%$ coverage and $>50 \%$ identity to proteins from Diptera downloaded from GenBank. Altogether, we submitted 1842 protein coding sequences to the Transcriptome Shotgun Assembly (TSA) portal of the NCBI. This procedure produced a final spreadsheet of 33,846 contigs (Additional file 4: Dataset S1, Additional file 5: Dataset S2, Additional file 6: Dataset S3, Additional file 7: Dataset S4 and Fig. 1) and allowed assigning a VectorBase ID code (AALFxxxxxx) to 14,386 transcriptome contigs and significantly reducing redundancy. Mapping the reads to the transcriptome revealed an overall acceptable correlation between replicates

Table 1 Summary of sequencing and assembly statistics

\begin{tabular}{ll}
\hline Female Antennae A (reads) & $29,945,374$ \\
\hline Female Antennae B (reads) & $17,712,800$ \\
Female Palps A (reads) & $7,698,073$ \\
Female Palps B (reads) & $5,354,526$ \\
Female Whole body A (reads) & $25,639,847$ \\
Female Whole body B (reads) & $19,011,547$ \\
Male Heads A (reads) & $8,457,785$ \\
Male Heads B (reads) & $19,688,382$ \\
Total bases (bp) & $133,508,334$ \\
Total assembled bases (bp) & $27,866,796$ \\
Assembled contigs & 33,846 \\
Average contig length (bp) & 823 \\
N50 (bp) & 1563 \\
Shortest transcript length (bp) & 150 \\
Longest transcript length (bp) & 32,463 \\
transcripts >1Kb & 8953 \\
transcripts > 2Kb & 3100 \\
\hline
\end{tabular}




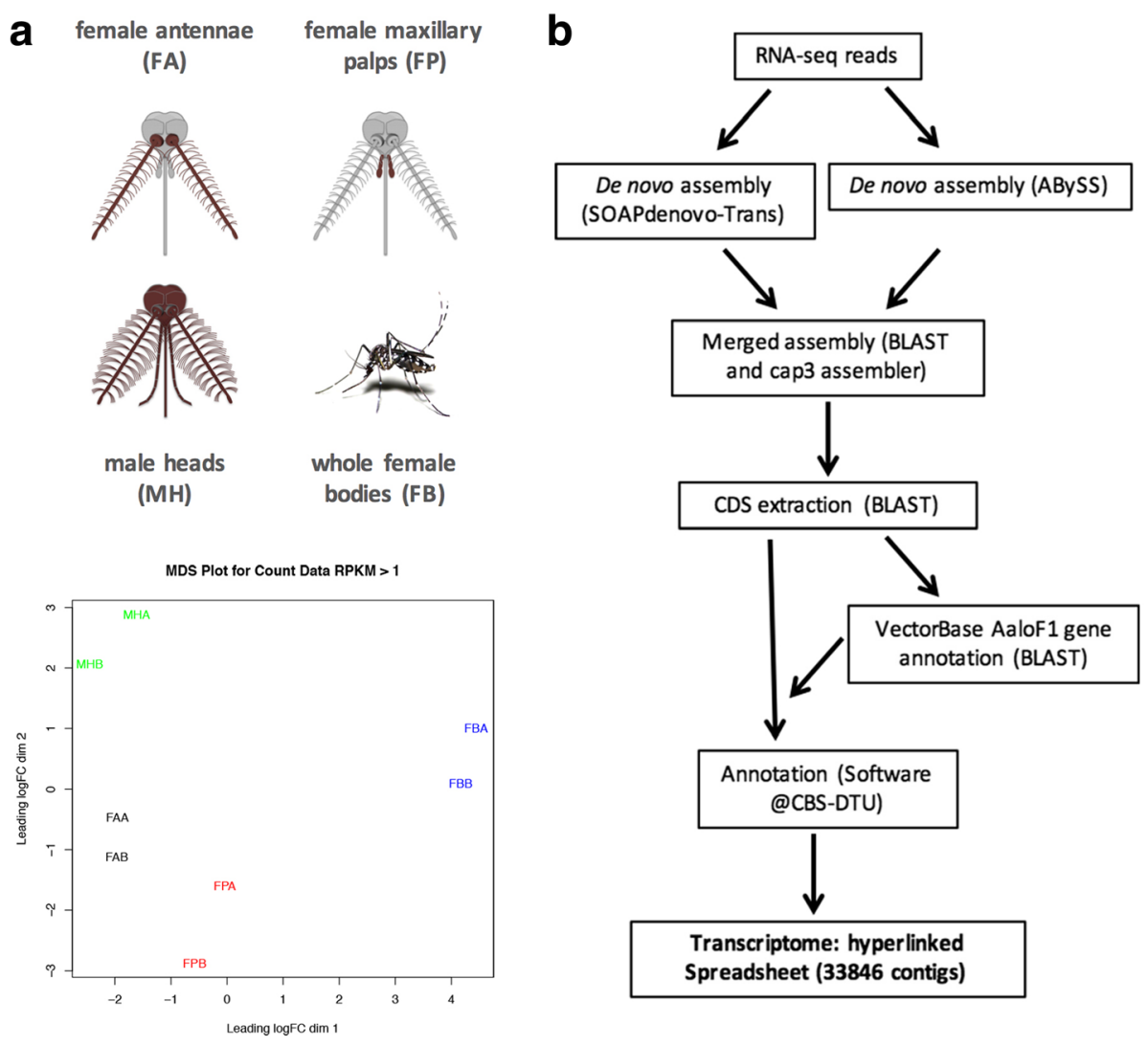

Fig. 1 Tissues, RNA-seq method and cluster analysis. a. Schematic representation of female head with highlighted in red antennae and palps (upper part of the panel), male head and whole female mosquito (lower part of the panel). b. Workflow chart of procedures used for the assembly and the annotation of the Ae. albopictus olfactory transcriptome. c. Multidimensional plot of RNA-seq duplicates used in this study

for each tissue (Fig. 1c). The maxillary palp samples of females showed the highest divergence (but still highly correlated) whereas, as expected, reads derived from head tissues (female antennae, FA; female palps, FP and male heads, $\mathrm{MH}$ ) displayed a higher correlation between them when compared to the female whole body (FB). Finally, a detailed cluster analysis was performed after assessment of TPM (transcripts per million) values for each replicate dataset (Additional file 8: Figure S1). This analysis confirmed the overall quality of replicates in our RNA-seq procedure and facilitated the identification of several groups of co-expressed genes that represent transcriptome profile signatures of the different tissues/sexes.

\section{Transcript abundance profiling in chemosensory tissues}

Tissue-specific transcript enrichment was evaluated by pairwise comparisons between samples. Fold change (FC) Logarithmic values and false discovery rates (FDR) were calculated using edgeR to provide a statistical validation (Additional file 6: Dataset S3). We selected transcripts (represented hereafter as genes) showing enhanced abundance in chemosensory tissues (female antennae, female palps and male heads) when compared to female whole body and compared them to each other (Figs. 2 and 3). A strict statistical FDR threshold $(P<0.001)$ was used to define subsets of highly enhanced genes in female antennae (620 contigs) and in female palps (268 contigs, Table 2 and Figs. 2 and 3. Within these criteria, 171 contigs were enriched in male heads as compared to female body, indicating a sex-biased abundance profile (Fig. 3). Previous

Table 2 Gene expression profiling by edgeR

\begin{tabular}{lllll}
\hline & & $P<0.05$ & $P<0.01$ & $P<0.001$ \\
\hline FA vs FB & FA UP: & $\mathbf{1 9 9 2}$ & $\mathbf{1 1 2 9}$ & $\mathbf{6 2 0}$ \\
& FB UP: & 3068 & 1962 & 1207 \\
& Total: & 5060 & 3091 & 1827 \\
FP vs FB & FP UP & $\mathbf{1 7 5 2}$ & $\mathbf{7 3 1}$ & $\mathbf{2 6 8}$ \\
& FB UP & 3445 & 1755 & 807 \\
& Total: & 5197 & 2486 & 1075 \\
MH vs FB & MH UP & $\mathbf{2 1 3 7}$ & $\mathbf{8 1 9}$ & $\mathbf{1 7 1}$ \\
& FB UP & 2918 & 1768 & 1091 \\
& Total: & 5055 & 2587 & 1262 \\
\hline
\end{tabular}

Table 2. Gene expression profiling by edgeR. Pairwise comparisons (FA vs FB, $\mathrm{FP}$ vs $\mathrm{FB}$ and $\mathrm{MH}$ vs $\mathrm{FB}$ ) highlight genes upregulated in each sample according to different probability thresholds $(P<0.05, P<0.01$ and $P<0.001)$ 


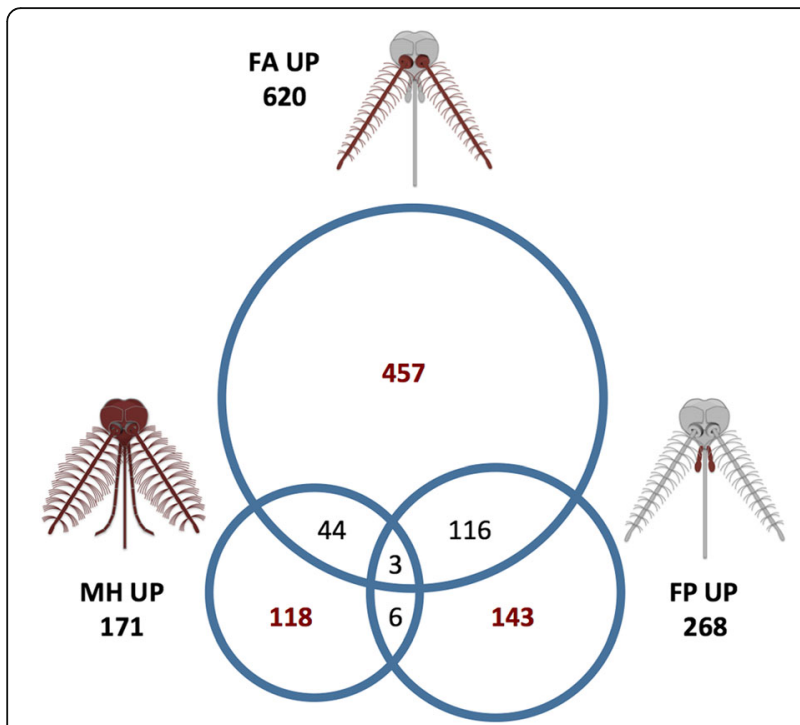

Fig. 2 Pairwise sample comparisons. Proportional Venn diagram showing pairwise comparisons between female antennae, female palps and male heads. Gene subsets enhanced in each sample versus female body according to the edgeR threshold at $P<0.001$ (see Table 2) were compared to each other. Overlaps (and relative numbers) represent the subsets of genes that are differentially expressed in more than one tissue

transcriptomic analysis in An gambiae and Ae. aegypti mosquitoes reported 2277 and 244 contigs differentially enhanced in female antennae, respectively [71, 74].

Evaluation of both PFAM and GO enrichment in the enhanced gene sets was performed (Tables 3-5 and Additional file 9: Figure S2). The PFAM analysis was performed selecting the gene sets identified by the restrictive FDR $<0.001$ threshold to increase the likelihood of isolating "tissue-specific" contigs. Not surprisingly, the three most frequent gene families in the antennae-enriched subset are $7 \mathrm{tm}$ Odorant receptor, PBP/GOBP and Ligand-gated ion channel (Table 3), similarly to previous observations in An. gambiae [74]. For the maxillary palps, the most significantly enhanced gene family comprises the ninjurin genes that are involved in several biological processes such as cell-cell, cell-matrix adhesion and nervous tissues development (Table 4). Other gene families enriched in the Ae. albopictus maxillary palps are the $7 \mathrm{tm}$ Chemosensory receptor (gustatory receptors), PBP/GOBP family and CD36 family that were also shown enriched in the maxillary palps of An. gambiae mosquitoes [74]. Finally, enriched gene families in male heads are represented mainly by Neurotransmitter-gated ion-channel ligand binding domain and 7 transmembrane receptor (rhodopsin family), since composed eyes and ganglia are large and abundant organs in this body part (Table 5).

Enrichment analysis of GO terms was performed comparing GO terms frequencies in tissue-specific subsets
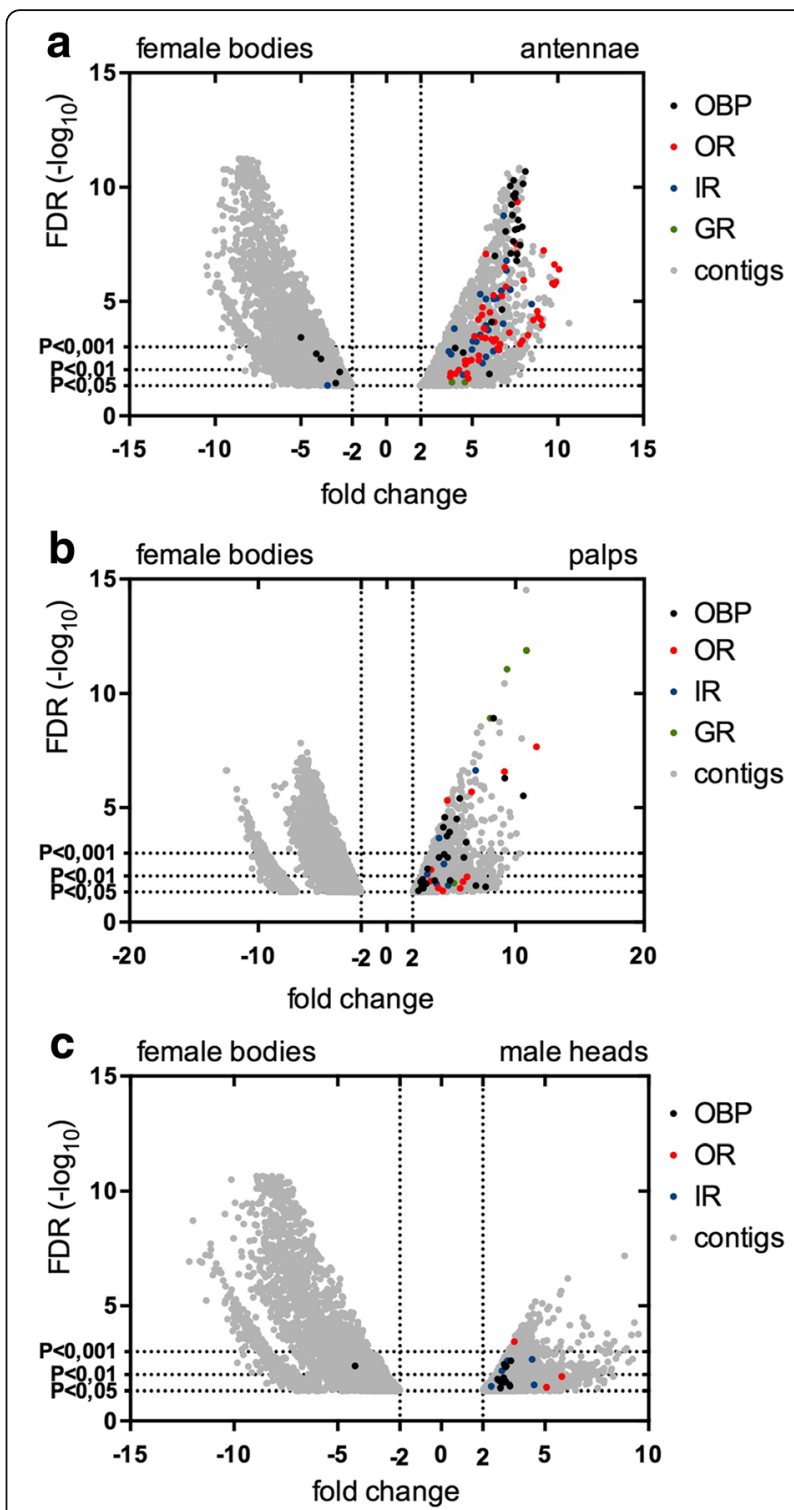

Fig. 3 Differential expression (DE) of chemosensory genes. Volcano plots show the relative expression of contigs in pairwise comparisons. The $x$-axis represents the logFC (fold change) between tissues. The $y$-axis represents the negative $\log 10$ of the $p$-value (false discovery rate) as calculated by the Fisher's Exact test. a. Female bodies vs Antennae.

b. Female bodies vs Palps. c. Female bodies vs Male Heads. Only differentially expressed contigs $(P<0.05, \log F C<-2$ and $>2)$ are shown in the plot (grey dots) with OBP indicated in black, $O R$ in red, $G R$ in green and IR in blue

$(P<0.01)$ with GO terms frequencies in the transcriptome. Statistically significant GO terms (cellular component, molecular function and biological process) are shown in Additional file 9: Figure S2. By this analysis, OBPs (in both antennae-UP and palps-UP specific subsets) as well as odorant and ionotropic glutamate receptors (in the antennae-UP subset) are among the most highly represented molecular functions. Structural 
Table 3 PFAM enrichment in Ae. albopictus female antennae vs transcriptome

\begin{tabular}{|c|c|c|c|c|c|}
\hline PFAM TERM & PFAM DESCRIPTION & N PFAM Transcriptome & $N^{\circ}$ PFAM FA UP 620 & $p$ value & FDR \\
\hline PF02949 & 7tm Odorant receptor & 77 & 35 & $3.74 \mathrm{E}-116$ & $1.62 \mathrm{E}-113$ \\
\hline PF01395 & PBP/GOBP family & 111 & 23 & $2.56 \mathrm{E}-38$ & $5.54 \mathrm{E}-36$ \\
\hline PF00060 & Ligand-gated ion channel & 45 & 14 & $1.50 \mathrm{E}-33$ & $2.16 \mathrm{E}-31$ \\
\hline PF03028 & Dynein heavy chain and region D6 of dynein motor & 16 & 6 & $5.12 \mathrm{E}-16$ & $5.54 \mathrm{E}-14$ \\
\hline PF03148 & Tektin family & 9 & 4 & $1.48 \mathrm{E}-11$ & $1.28 \mathrm{E}-09$ \\
\hline PF00025 & ADP ribosylation factor & 17 & 4 & $3.92 \mathrm{E}-07$ & $1.70 \mathrm{E}-05$ \\
\hline PF04923 & Ninjurin & 17 & 4 & $3.92 \mathrm{E}-07$ & $1.70 \mathrm{E}-05$ \\
\hline PF13414 & TPR repeat & 29 & 5 & $6.95 \mathrm{E}-07$ & 2.74E-05 \\
\hline PF00043 & Glutathione S-transferase. C-terminal domain & 12 & 3 & $1.81 \mathrm{E}-05$ & 0.00052 \\
\hline PF00250 & Forkhead domain & 18 & 3 & 0.00057 & 0.01179 \\
\hline PF04415 & Protein of unknown function (DUF515) & 19 & 3 & 0.00084 & 0.01671 \\
\hline PF02497 & Arterivirus glycoprotein & 7 & 2 & 0.00089 & 0.01692 \\
\hline PF03392 & Insect pheromone-binding family. A10/OS-D & 20 & 3 & 0.00121 & 0.02198 \\
\hline
\end{tabular}

Table 3. PFAM enrichment in Ae. albopictus female antennae vs transcriptome. Summary of Protein families statistically overrepresented in tissue-enriched subset, FA-UP

features such as dynein complex (cellular component) and microtubule-based movement and cilium assembly (biological process) are also significantly represented among antennae-specific GO terms.

Ae. albopictus antennae and palps are not only distinguishable as a result of significant differences in both PFAM and GO enrichment analyses but also when looking at specific and shared transcripts (i.e., contigs specific to the antennae, to the palps and to male heads as well as those whose expression was enhanced in more than one chemosensory appendage). These relationships are represented in a proportional Venn diagram (Fig. 2) that highlights the overlaps between tissues (antennae and palps) as well as tissue- and sex-specific groups. Comparison between the subset of female antennaeenriched contigs with those enriched in palps revealed a relevant overlap, with 119 enriched transcripts (44\% of palp set) in common (Fig. 2). Around $28 \%$ of transcripts enhanced in male heads are also enhanced in the female antennae, while only $5 \%$ of palps-specific contigs are enhanced in male head. This comparison also identified groups of transcripts specific to the female antennae (457/620, 73\%), female palps $(143 / 268,53 \%)$ and male heads $(118 / 171,69 \%)$.

\section{Chemosensory gene families}

Chemosensory pathways in vector mosquitoes have been extensively characterized through genomics and transcriptomics studies in the last 10 years providing the scientific community with comprehensive lists of olfactory genes from An. gambiae, Ae. aegypti and C. quinquefasciatus [38, 69-71, 74-77]. We have searched the

Table 4 PFAM enrichment in Ae. albopictus female maxillary palps vs transcriptome

\begin{tabular}{|c|c|c|c|c|c|}
\hline PFAM TERM & PFAM DESCRIPTION & Nº PFAM Transcriptome & No PFAM FP UP 268 & $\mathrm{p}$ value & FDR \\
\hline PF04923 & Ninjurin & 17 & 4 & 2.67E-16 & 1.32E-14 \\
\hline PF08395 & 7tm Chemosensory receptor & 33 & 5 & $1.76 \mathrm{E}-14$ & $6.96 \mathrm{E}-13$ \\
\hline PF01395 & PBP/GOBP family & 111 & 8 & 1.27E-11 & 4.19E-10 \\
\hline PF01130 & CD36 family & 17 & 3 & 3.81E-09 & $1.08 \mathrm{E}-07$ \\
\hline PF00650 & CRAL/TRIO domain & 40 & 4 & 8.93E-08 & 2.21E-06 \\
\hline PF06585 & Haemolymph juvenile hormone binding protein (JHBP) & 26 & 3 & 2.10E-06 & 3.77E-05 \\
\hline PF00188 & Cysteine-rich secretory protein family & 29 & 3 & 7.86E-06 & 0.00012 \\
\hline PF00106 & short chain dehydrogenase & 71 & 4 & 0.00015 & 0.00095 \\
\hline PF02958 & Ecdysteroid kinase & 51 & 3 & 0.00150 & 0.00663 \\
\hline PF00135 & Carboxylesterase family & 66 & 3 & 0.00815 & 0.02086 \\
\hline PF02949 & $7 \mathrm{tm}$ Odorant receptor & 77 & 3 & 0.01891 & 0.04150 \\
\hline
\end{tabular}

Table 4. PFAM enrichment in Ae. albopictus female maxillary palps vs transcriptome. Summary of Protein families statistically overrepresented in tissue-enriched subset, FP-UP 
Table 5 PFAM enrichment in Ae. albopictus male heads vs transcriptome

\begin{tabular}{|c|c|c|c|c|c|}
\hline PFAM TERM & PFAM DESCRIPTION & $\mathrm{N}^{\circ}$ PFAM Transcriptome & N PFAM MH UP 171 & $p$ value & FDR \\
\hline PF02931 & Neurotransmitter-gated ion-channel ligand binding domain & 29 & 3 & $5.56 \mathrm{E}-09$ & 2.06E-07 \\
\hline PF00001 & 7 transmembrane receptor (rhodopsin family) & 81 & 4 & $2.60 \mathrm{E}-06$ & $1.48 \mathrm{E}-05$ \\
\hline PF00400 & WD domain. G-beta repeat & 86 & 3 & 0.00216 & 0.00533 \\
\hline
\end{tabular}

Table 5. PFAM enrichment in Ae. albopictus male heads vs transcriptome. Summary of Protein families statistically overrepresented in tissue-enriched subset, MH-UP

transcriptome profiles for genes with products that are involved in sensory functions by blasting the contigs against several databases (as, for instance, Invertebrate and Diptera protein databases), as detailed in the Methods section. We have also used published datasets of Ae. aegypti chemosensory genes as a query (ORs: [69-71]; GRs [22, 69, 71]; IRs: [27, 69, 71]; OBPs: $[38,69,71]$ ) and a reciprocal BLASTP algorithm to identify true orthologs in our Ae. albopictus transcriptome. Finally, the lists were further extended by manually guided reciprocal BLAST that allowed including contigs showing high similarity over shorter regions. Lists of 77 OBPs, 82 ORs, 60 IRs and 30 GRs were obtained (Table 6 and Figs. 4-7). As detailed below, several members of each family showed specific or enriched abundance in one or both olfactory appendages (Additional file 10: Table S1 and Figs. 3-7).

\section{Odorant binding proteins}

Taken together our RNA-seq analysis indicates that $A e$. albopictus transcriptome includes at least 77 OBPs carrying amino acid signatures characteristic of several different OBP sub-families and clusters as described in [38] (Fig. 4, Additional file 11: Figure S3, Additional file 12: Figure S4, Additional file 13: Figure S5, Additional file 14: Figure S6). Accordingly, these could be classified as Classic OBPs (32), PlusC OBPs (16) and Atypical OBPs (26). In addition, 3 OBPs could be classified as MinusC, lacking cysteines $\mathrm{C} 2$ and C5 (Fig. 4). The evolution of the MinusC subfamily in Holometabola is quite intriguing, since members of this sub-group were found in the Drosophilidae, Bombyx/ Tribolium, and Apis lineages as well as in the genomes of Ae. aegypti and C. quinquefasciatus but not in An. gambiae [38]. A possible link to the Atypical/Two domains subfamily is provided by the observation that the "matype2" group (name of this OBP cluster as defined in [38]) is characterized by a first OBP-like domain lacking $\mathrm{C} 2$ and $\mathrm{C} 5$ cysteines [38]. Four of the putative OBP proteins identified (AalbOBP20/59-AALF005057, AalbOBP25/24-AALF018602, AalbOBP26/23-AALF018601 and AalbOBP63/42-Ae2SigPSigP-217188_FR2_96-312) show the same percentage of identity with multiple $A e$. aegypti OBPs. Moreover, two contigs (AALF022642 and AALF020568) showing high sequence similarity with OBP domains were unusually long and therefore have been manually annotated. They were probably chimeric genes wrongly built during the assembly step: the novel putative sequences are reported in Additional file 15: Dataset S5.

The intensity range of OBP transcript abundance is broad, spanning from 0 to 33,100 TPM. As expected, most transcripts encoding Classic OBPs displayed enhanced abundance in female antennae (heat map in Fig. 4), while PlusC family members showed a less defined profile with some OBP specifically enhanced in female palps (e.g. AalbOBP51 and AalbOBP70). Atypical (Two domains) OBPs were generally expressed at low levels in all samples analysed, with the only exception of AalbOBP107, which is the only Atypical OBP with $\mathrm{TPM}>1$. Classic and PlusC OBP transcripts were also robustly abundant in male heads. Although our dataset does not include female heads, which would give indication regarding non-chemosensory neuronal tissues, it is likely that at least some of these proteins (especially belonging to the Classic sub-family) may be involved in physiological processes and perhaps behaviours shared between the two sexes (Additional file 10: Table S1). Among contigs enhanced in the antennae, AALF023075 encodes for the putative homolog of An. gambiae OBP1 (AGAP003309, 78\% identity) that was shown to mediate the binding with the attractant indole [41]. Recent

Table 6 Chemosensory gene families

\begin{tabular}{lllll}
\hline & Transcriptome (33,846) & Antennae (5060) & Palps (5197) & Male Heads (5055) \\
\hline OBP & $77(68)$ & 30 & 26 & 15 \\
OR & $82(59)$ & 52 & 11 & 3 \\
IR & $60(43)$ & 25 & 6 & 6 \\
GR & $30(28)$ & 2 & 4 & 0 \\
Tot. & 249 & 109 & 47 & 24 \\
\hline
\end{tabular}

Table 6. Chemosensory gene families. In the column Transcriptome is reported the number of genes belonging to each chemosensory family identified in the transcriptome upon manual and automated (in brackets) BLAST analysis. In the other columns (Antennae, Palps and Male Heads) are reported the number of chemosensory genes in each enriched subset (see Table 2, threshold at FDR $<0.05$ ) 


\begin{tabular}{|c|c|c|c|c|c|c|c|c|c|}
\hline FA & FP & MH & FB & OBP name & Ae. albopictus ID & \begin{tabular}{|l|l|} 
Cys & Subfamily \\
\end{tabular} & OBP name (Ae. aegypti) & Ae. aegypti ID & $\%$ \\
\hline & & & & AalbOBP57 & AALF021771 & 7 classic & AaegOBP57 & AAEL000035 & 80 \\
\hline & & & & AalbOBP56 & AALF026945 & 8 classic & AaegOBP56 & AAEL000051 & 90 \\
\hline & & & & AalbOBP27 & Ae2SigPSigP-218581_FR4_17-171 & 6 classic & AaegOBP27 & AAEL000071 & 85 \\
\hline & & & & AalbOBP4 & Ae2SigPSigP-245019_FR5_303-456 & 6 classic & AaegOBP4 & AAEL000073 & 92 \\
\hline & & & & AalbOBP11 & AALF007517 & 6 classic & AaegOBP11 & AAEL002587 & 84 \\
\hline & & & & AalbOBP13 & AALF019175 & 7 classic & AaegOBP13 & AAEL002591 & 89 \\
\hline & & & & AalbOBP9 & AALF024996 & 6 classic & AaegOBP9 & AAEL002596 & 84 \\
\hline & & & & AalbOBP15 & AALF019178 & 7 classic & AaegOBP15 & AAEL002598 & 97 \\
\hline & & & & AalbOBP35 & AALF013191 & 6 classic & AaegOBP35 & AAEL002606 & 100 \\
\hline & & & & AalbOBP12 & AALF019174 & 7 classic & AaegOBP12 & AAEL002617 & 87 \\
\hline & & & & AalbOBP65 & AALF019177 & 6 classic & AaegOBP65 & AAEL002618 & 91 \\
\hline & & & & AalbOBP17 & Ae2SigPSigP-70660_FR2_3-125 & 3 classic & AaegOBP17 & AAEL004339 & 74 \\
\hline & & & & AalbOBP18 & Ae2SigPSigP-221527_FR6_753-897 & 8 classic & AaegOBP18 & AAEL004342 & 80 \\
\hline & & & & AalbOBP19 & AALF023074 & 4 classic & AaegOBP19 & AAEL004343 & 74 \\
\hline & & & & AalbOBP21 & AALF014342 & 14 classic & AaegOBP21 & AAEL005770 & 89 \\
\hline & & & & AalbOBP22 & AALF014341 & 6 classic & AaegOBP22 & AAEL005772 & 93 \\
\hline & & & & AalbOBP20/59 & AALF005057 & 7 classic & AaegOBP20/59 & AAEL005778/AAEL015313 & $87 / 87$ \\
\hline & & & & AalbOBP2 & Ae2SigPSigP-245707_FR2_11-195 & 8 classic & AaegOBP2 & AAEL006176 & 95 \\
\hline & & & & AalbOBP39 & AALF005090 & 6 classic & AaegOBP39 & AAEL006454 & 92 \\
\hline & & & & AalbOBP80 & AALF015707 & 6 classic & AaegOBP80 & AAEL007003 & 74 \\
\hline & & & & AalbOBP79 & AALF022738 & 7 classic & AaegOBP79 & AAEL007014 & 80 \\
\hline & & & & AalbOBP10 & AALF027078 & 7 classic & AaegOBP10 & AAEL007603 & 82 \\
\hline & & & & AalbOBP37 & Ae2SigPSigP-225473_FR6_9-169 & 8 classic & AaegOBP37 & AAEL008009 & 89 \\
\hline & & & & AalbOBP36 & AALF009587 & 9 classic & AaegOBP36 & AAEL008011 & 84 \\
\hline & & & & AalbOBP38 & Ae2SigPSigP-224714_FR1_155-257 & 4 classic & AaegOBP38 & AAEL008013 & 100 \\
\hline & & & & AalbOBP1 & AALF023075 & 11 classic & AaegOBP1 & AAEL009449 & 93 \\
\hline & & & & AalbOBP83 & AALF018890 & 8 classic & AaegOBP83 & AAEL011416 & 87 \\
\hline & & & & AalbOBP81 & AALF007787 & 6 classic & AaegOBP81 & AAEL011730 & 78 \\
\hline & & & & AalbOBP55 & AALF021480 & 7 classic & AaegOBP55 & AAEL012377 & 92 \\
\hline & & & & AalbOBP3 & Ae2SigPSigP-246894_FR3_61-234 & 7 classic & AaegOBP3 & AAEL013018 & 94 \\
\hline & & & & AalbOBP34 & AALF028521 & 6 classic & AaegOBP34 & AAEL014082 & 88 \\
\hline & & & & AalbOBP60 & AALF002302 & 7 classic & AaegOBP60 & AAEL015499 & 93 \\
\hline & & & & AalbOBP78 & AALF008469 & 5 minus $C$ & AaegOBP78 & AAEL001836 & 65 \\
\hline & & & & AalbOBP77 & AALF024997 & 5 minus $C$ & AaegOBP77 & AAEL002626 & 92 \\
\hline & & & & AalbOBP76 & Ae2SigPSigP-244899_FR6_5-155 & 5 minus $C$ & AaegOBP76 & AAEL007604 & 85 \\
\hline & & & & AalbOBP69 & Ae2SigPSigP-30328_FR1_5-140 & 5 plus $\mathrm{C}$ & AaegOBP69 & AAEL000124 & 60 \\
\hline & & & & AalbOBP5 & AALF026669 & 13 plus $\mathrm{C}$ & AaegOBP5 & AAEL000139 & 88 \\
\hline & & & & AalbOBP72 & AALF013104 & 12 plus $\mathrm{C}$ & AaegOBP72 & AAEL004729 & 78 \\
\hline & & & & AalbOBP73 & Ae2SigPSigP-31227_FR4_1-172 & 12 plus $\mathrm{C}$ & AaegOBP73 & AAEL004730 & 72 \\
\hline & & & & AalbOBP25/24 & AALF018602 & 10 plus $\mathrm{C}$ & AaegOBP25/24 & AAEL006103/AAEL006108 & $95 / 95$ \\
\hline & & & & AalbOBP48 & AALF018600 & 12 plus $\mathrm{C}$ & AaegOBP70 & AAEL006105 & 86 \\
\hline & & & & AalbOBP26/23 & AALF018601 & 14 plus $\mathrm{C}$ & AaegOBP26/23 & AAEL006106/AAEL006109 & $87 / 87$ \\
\hline & & & & AalbOBP54 & AALF020570 & 12 plus $\mathrm{C}$ & AaegOBP54 & AAEL011481 & 67 \\
\hline & & & & AalbOBP75 & AALF020567 & 12 plus $\mathrm{C}$ & AaegOBP75 & AAEL011483 & 81 \\
\hline & & & & AalbOBP49 & AALF020568 & 12 plus $\mathrm{C}$ & AaegOBP49 & AAEL011484 & 89 \\
\hline & & & & AalbOBP74 & Ae2SigPSigP-29640_FR2_1-211 & 12 plus $\mathrm{C}$ & AaegOBP74 & AAEL011486 & 90 \\
\hline & & & & AalbOBP51 & AALF020569 & 13 plus $\mathrm{C}$ & AaegOBP51 & AAEL011487 & 76 \\
\hline & & & & AalbOBP70 & AALF020565 & 11 plus $C$ & AaegOBP48 & AAEL011494 & 85 \\
\hline & & & & AalbOBP47 & Ae2SigPSigP-237436_FR3_27-219 & 15 plus $\mathrm{C}$ & AaegOBP47 & AAEL011499 & 83 \\
\hline & & & & AalbOBP62 & AALF001353 & 5 plus $\mathrm{C}$ & AaegOBP62 & AAEL015566 & 84 \\
\hline & & & & AalbOBP63/42 & Ae2SigPSigP-217188_FR2_96-312 & 13 plus $\mathrm{C}$ & AaegOBP63/42 & AAEL015567/AAEL010666 & $97 / 97$ \\
\hline & & & & AalbOBP90 & AALF008134 & 6 atypical & AaegOBP90 & AAEL013719 & 83 \\
\hline & & & & AalbOBP89 & AALF006399 & 13 two domains & AaegOBP89 & AAEL000377 & 72 \\
\hline & & & & AalbOBP97 & AALF017937 & 15 two domains & AaegOBP97 & AAEL000835 & 78 \\
\hline & & & & AalbOBP112 & AALF017939 & 15 two domains & AaegOBP112 & AAEL000837 & 73 \\
\hline & & & & AalbOBP7-N1 & AALF017938 & 15 two domains & AaegOBP7-N1 & AAEL000846 & 74 \\
\hline & & & & AalbOBP106 & AALF013134 & 11 two domains & AaegOBP106 & AAEL001153 & 68 \\
\hline & & & & AalbOBP98 & AALF013138 & 14 two domains & AaegOBP98 & AAEL001174 & 81 \\
\hline & & & & AalbOBP105 & AALF009317 & 17 two domains & AaegOBP105 & AAEL001189 & 77 \\
\hline & & & & AalbOBP111-N1 & AALF003184 & 15 two domains & AaegOBP111 & AAEL003311 & 64 \\
\hline & & & & AalbOBP111 & AALF003679 & 14 two domains & AaegOBP111 & AAEL003311 & 66 \\
\hline & & & & AalbOBP16 & AALF018393 & 17 two domains & AaegOBP16 & AAEL003315 & 77 \\
\hline & & & & AalbOBP100 & AALF017970 & 18 two domains & AaegOBP100 & AAEL003513 & 61 \\
\hline & & & & AalbOBP101 & AALF010900 & 17 two domains & AaegOBP101 & AAEL003525 & 72 \\
\hline & & & & AalbOBP102 & AALF011068 & 18 two domains & AaegOBP102 & AAEL003538 & 60 \\
\hline & & & & AalbOBP104 & AALF013136 & 14 two domains & AaegOBP104 & AAEL004516 & 78 \\
\hline & & & & AalbOBP33 & AALF022642 & 16 two domains & AaegOBP33 & AAEL006385 & 90 \\
\hline & & & & AalbOBP28 & AALF022644 & 19 two domains & AaegOBP28 & AAEL006393 & 91 \\
\hline & & & & AalbOBP113 & AALF000521 & 11 two domains & AaegOBP113 & AAEL008640 & 80 \\
\hline & & & & AalbOBP40 & AALF008889 & 14 two domains & AaegOBP40 & AAEL009597 & 85 \\
\hline & & & & AalbOBP44 & AALF015687 & 17 two domains & AaegOBP44 & AAEL010718 & 54 \\
\hline & & & & AalbOBP46 & AALF013004 & 19 two domains & AaegOBP46 & AAEL010872 & 80 \\
\hline & & & & AalbOBP103 & AALF013003 & 15 two domains & AaegOBP103 & AAEL010875 & 69 \\
\hline & & & & AalbOBP58 & AALF021668 & 12 two domains & AaegOBP58 & AAEL014430 & 84 \\
\hline & & & & AalbOBP110 & AALF021669 & 13 two domains & AaegOBP110 & AAEL014431 & 88 \\
\hline & & & & AalbOBP108 & AALF014080 & 12 two domains & AaegOBP108 & AAEL014874 & 71 \\
\hline & & & & AalbOBP107 & AALF014081 & 13 two domains & AaegOBP107 & AAEL014876 & 73 \\
\hline
\end{tabular}

percentiles

$10 \quad 50 \quad 90$

Fig. 4 (See legend on next page.) 
(See figure on previous page.)

Fig. 4 Odorant binding proteins (OBPs) in Ae. albopictus transcriptome. Left panel, abundance profile map: intensity scale (color gradient from blue to yellow indicates levels from high TPM to low) as indicated at the bottom. FA, female antennae; FP, female palps; MH male heads and FB, female body. Assigned OBP names, ID in Ae. albopictus (VectorBase ID AALFxxxxxx when available or contig ID in the transcriptome), number of cysteines, OBP subfamily, name and ID of the presumed Ae. aegypti ortholog and percentage of identity are reported

functional studies based on the analysis of binding affinities for physiologically relevant compounds showed a certain degree of selectivity for a few mosquito OBPs, able to preferentially carry certain ligands than others $[39,41,78]$. Taken together, the transcriptome profiles presented here are consistent with previously published transcriptomic datasets of Ae. aegypti palps [69] and antennae [71, 79]. A total of 28 OBPs were previously described in Ae. albopictus [80-82] and are summarized in Additional file 16: Table S2. A comprehensive list of Ae. albopictus putative OBP (86) was predicted from the genome sequence and validated by RNA-seq analysis of developmental stages [62]: a detailed comparison with OBP from our transcriptome is reported in Additional file 17: Table S3.

\section{Odorant receptors}

Our analysis identified 82 contigs encoding putative ORs in the combined transcriptome profile of Ae. albopictus chemosensory tissues. Within a threshold of TPM $>3$ in at least one sample, 49 OR transcripts may be classified into three broad categories. Most ORs (39) are enriched in the female antennae (using as sorting criteria, those with TPM $>3$ in the antennae, TPM $<3$ in the other tissues and at least 10-fold TPM increase in the antennae compared to palps and/or male heads). Among these, the homologue (79\% identity) of AaegOR4 was identified in Ae. albopictus and showed a comparable intensity and specificity of expression in the female antennae [71, 79]. Recent studies in Ae. aegypti found AaegOR4 enriched in the antennae of Ae. aegypti mosquitoes with an anthropophilic host preference therefore suggesting this OR could play an important role in establishing that important behavior [19]. A second group comprising 6 ORs displayed a more ubiquitous abundance profile and, not surprisingly, this includes the transcript for the obligate ORco co-receptor that is expressed in all ORNs where it is required for establishing functional ORcomplexes [6]. Finally, three ORs (AalbOR8, AalbOR49 and AalbOR91) are highly enriched if not specific to Ae. albopictus maxillary palps Fig. 3 and Fig. 5, which aligns to similar patterns already described for their orthologs in An. gambiae [24, 83] and Ae. aegypti [69]. Indeed, homologues of AalbOR8 show enhanced expression profiles in Ae. aegypti and An. gambiae maxillary palps, as the homologue of AalbOR49 in Ae. aegypti is similarly enriched in the palps $[24,69]$. In An. gambiae AgOR8 is expressed in maxillary palp OSNs that are highly sensitive to 1-octen-3-ol [24], a chemical component contained in human breath and sweat, one of the factors responsible for the attraction to human host. Interestingly, Ae. albopictus mosquitoes are similarly attracted by 1 -octen-3-ol [84].

Beyond these, 25 contigs encode putative ORs that display low sequence identity $(<70 \%)$ with cognate $A e$. aegypti orthologs; some of these may be novel Ae. albopictus receptors resulting from gene duplication and subsequent evolutionary divergence. As in Anophelines [85], the rapid gain (and loss) of ORs that belong to chromosomal gene clusters is a hallmark of speciesspecific evolution. This can be inferred especially when multiple putative Ae. albopictus orthologs (with more than $\sim 70 \%$ identity) are found by BLASTP searches using the same Ae aegypti OR as query, and quite a few examples (9 AAELxxxxxx queries match with more than one AALFxxxxx/contig) can be found in Fig. 5. Among these, Ae. aegypti OR61 (AAEL017277) identifies 4 putative homologs in the Ae. albopictus transcriptome (Additional file 18: Figure S7). However, only AALF018937/AalbOR61 was relatively abundant $(5<\mathrm{TPM}<80)$ in all samples analysed and may be considered the true ortholog of AaegOR61 also in view of the higher identity (79\%). The remaining three (AALF018936/AalbOR61-N1, AALF003919/AalbOR61-N2 and AALF003920/AalbOR61-N3) are most likely the result of gene duplication, show lower transcript abundance $(5<\mathrm{TPM})$ in all samples and their possible role needs further investigation. A similar situation is found for AaegOR70 (AAEL001224) with two hits in the Ae. albopictus transcriptome: (i) Ae2SigPSigP-43314_FR6_155-284/AalbOR70, which is rather abundant in all samples and shows higher identity (79\%) to AaegOR70 and (ii) AALF019061/AalbOR70-N1, which is enriched in female antennae and shares 64\% identity with AaegOR70.

Further studies will be necessary to validate the hypothesis that gene duplication events took place in the Ae. albopictus OR gene families. Antennal transcriptomic analysis of two sibling Anophelinae species with different feeding behaviour showed that while their OR repertoires were highly conserved, the rates of evolution of each chemosensory gene family were more rapid than the genomic background and, importantly, there were species-specific shifts in OR transcriptome profiles [86]. Moreover, while there is an overall conservation in OR gene numbers among 16 anophelinae species, probably because of their role in several critical behaviours, 


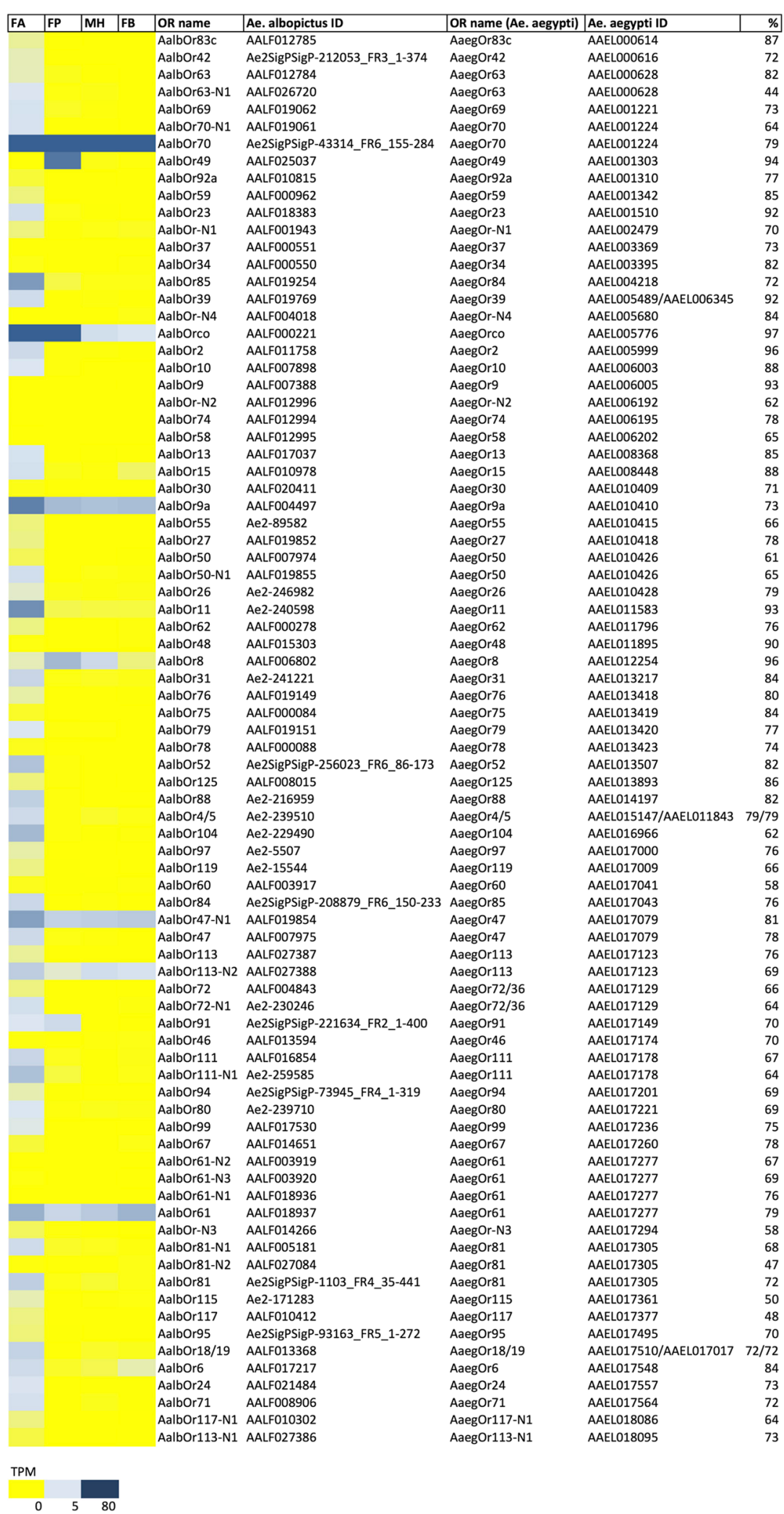

Fig. 5 (See legend on next page.) 
(See figure on previous page.)

Fig. 5 Odorant receptors (OR) in Ae. albopictus transcriptome. Left panel, abundance profile map: intensity scale (color gradient from blue to yellow indicates levels from high TPM to low) as indicated at the bottom. FA, female antennae; FP, female palps; MH male heads and FB, female body. OR name and Ae. albopictus ID columns indicate respectively the name assigned to OR in Ae. albopictus and the ID (VectorBase ID AALFxxxxxx when available or contig ID in the transcriptome). Ae. aegypti OR name ID columns list the OR name in Ae. aegypti and the VectorBase code (AAELxxxxxx) of Ae. aegypti orthologs, respectively, with the percent (\%) of identity reported in the last column

numerous examples of gene gain and loss in specific anophelinae lineages were noted, reflecting the importance of ORs in the functional divergence and acquisition of novel features in the evolution of different behavioural traits (feeding behaviour, mating, oviposition site choice, etc.) [85]. A few Ae. albopictus ORs have been previously characterized: the female antennae-specific AalOR2 [87] renamed as AalbOR2 in this study, the Ae. albopictus ORco co-receptor and a subset of tuning AalbORs already published [88]. Moreover, a list of predicted OR was deduced from the genome sequence and validated by RNA-seq analysis of developmental stages [62]: the comparison with OR described in our transcriptome is shown in Additional file 17: Table S3.

\section{Ionotropic receptors}

Sixty contigs encoding putative Ae. albopictus IRs (AalbIRs) were identified, most of which enriched in female antennae (Fig. 3 and Fig. 6). Setting the threshold at TPM > 3 in at least one tissue, 29 putative AalbIRs could be identified. Among these 15 AalbIRs are either specific to the female antennae or display at least a 10-fold increase in raw TPM when compared to male heads and female palps. A second group of at least 8 AalbIRs showed similar abundance in most if not all samples analysed and, not surprisingly, they include the Ae. albopictus orthologs of IR25a and IR8a which were shown to act as IR co-receptors in both Drosophila [28, 89-91] and more recently $A n$. gambiae (Pitts et al. 2017). It is worth noticing that IR25a and IR8a transcripts are highly abundant in antennae and/or maxillary palps of Ae. albopictus, Ae. aegypti and An gambiae mosquitoes [69, 71, 83]; instead in Culex quinquefasciatus while IR8a was found the most abundant IR expressed in the antennae [92], IR25a was absent [93]. Finally, AalbIR76b, which is the homologue of the third IR co-receptor IR76b, recently shown to be required to detect the smell and taste of polyamines in the fruit fly [31], is only moderately abundant in the Ae. albopictus female antennae. In contrast, IR76b is expressed at high levels in both $A n$. gambiae antennae and maxillary palps [74, 94], in the antennae, palps and labellum of Ae aegypti [69, 71, 79], as well as is found enriched in the antennae of $C$. quinquefasciatus [93].

Beyond these co-receptors, thirteen members of the IR41 sub-family of receptors are found in the transcriptome, with most of them (11/13) showing an enriched presence in the antennae sample. IR41a and IR41c have been recently shown to bind the heterocyclic amine pyrrolidine in An. gambiae [29]. Moreover, studies in the fruit fly demonstrated that IR41a, co-expressed with IR76b, mediates odour attraction, detecting polyamines present in fruits and in specific oviposition sites [31]. Interestingly, polyamines are also responsible for the attraction of Ae. aegypti mosquitoes to egg-laying sites [31].Two putative orthologs of AgIR41a are found in Ae. albopictus, AALF009939 (AalbIR41a.1) and Ae2248,662 (AalbIR41a.2) showing 56\% and 44\% identity, respectively. Another possible gene duplication event may be described for IR41p.1, which is encoded by two genes in Ae. aegypti: AAEL000063 and AAEL018060. In Ae. albopictus, AalbIR41p.1 (AALF024315) shows a good level (77\%) of identity with Ae. aegypti AAEL000063 (Fig. 6 and Additional file 19: Figure S8) and presents a weak expression profile (only in male heads TPM >1). AAEL018060 has two putative orthologs in Ae. albopictus, showing different expression profiles: AALF009945 (AalbIR41-like1) that is specifically expressed only in female antennae (TPM $=8,82$ and $\mathrm{TPM}<1$ in the other tissues) and AALF026946 (AalbIR41-like2) that is specific of both female antennae and palps. Other putative gene duplication events were found while using the $A e$. aegypti AaegIR111.2 (AAEL003249) as query to search our transcriptome. Indeed, five Ae. albopictus homologs (58 to $66 \%$ identity) expressed at low abundance in olfactory organs $(\mathrm{TPM}<1)$ and four of which arranged in cluster were identified in our olfactory transcriptome (Fig. 6, Additional file 20: Figure S9B). Moreover, as shown in Additional file 20: Figure S9A (from VectorBase), ten putative Ae. albopictus homologues of AaegIR111.2 and of the 75\% identical AAEL018094 are predicted by the Comparative Gene Tree tool in VectorBase (VBGT00730000019944). Finally, another example of genetic cluster rearrangement was analysed in detail for IR7. In Ae. albopictus, at least 10 contigs belonging to the IR7 family were found. They show a generally weak expression profile; seven of these are clustered in a $\sim 50 \mathrm{~kb}$ region and careful analysis indicated that two additional family members with no evidence of expression may be part of the same cluster (Additional file 21: Figure S10). Comparison to the Ae. aegypti genomic scaffold containing the IR7 cluster reveals a certain degree of gene shuffling among family members previously annotated in Ae. aegypti, with six orthologs identified in 


\begin{tabular}{|c|c|c|c|c|c|c|c|c|}
\hline FA & FP & MH & FB & IR name & Ae. albopictus ID & IR name (Ae. aegypti) & Ae. aegypti ID & $\%$ \\
\hline & & & & Aalblr41a.1 & AALF009939 & AaegIr41a.1 & AAEL000007 & 83 \\
\hline & & & & Aalblr41e & AALF024313 & Aaeglr41e & AAEL000011 & 70 \\
\hline & & & & Aalbılr41j & AALF009943 & Aaeglr41j & AAEL000018 & 71 \\
\hline & & & & Aalblr41d.1 & AALF021769 & AaegIr41d.1 & AAEL000027 & 85 \\
\hline & & & & Aalblr41a.2 & Ae2-248662 & Aaeglr41a.2 & AAEL000031 & 79 \\
\hline & & & & Aalblr41i & AALF009940 & AaegIr41i & AAEL000047 & 75 \\
\hline & & & & Aalblr41p.1 & AALF024315 & AaegIr41p.1 & AAEL000063 & 77 \\
\hline & & & & Aalblr41k & AALF009942 & Aaeglr41k & AAEL000066 & 79 \\
\hline & & & & Aalblr41n & Ae2SigPSigP-238240_FR4_1-432 & Aaeg|r41n & AAEL000089 & 88 \\
\hline & & & & Aalblr41d.3 & AALF021770 & AaegIr41d.3 & AAEL000095 & 90 \\
\hline & & & & Aalblr7j & AALF019121 & AaegIr7j & AAEL000413 & 71 \\
\hline & & & & Aalbır7l & AALF019122 & AaegIr7| & AAEL000462 & 70 \\
\hline & & & & AalbGlur5-6-7 & AALF021686 & AaegGlur5-6-7 & AAEL002506 & 97 \\
\hline & & & & AalbGlur5-6-7 & AALF026077 & AaegGlur5-6-7 & AAEL002509 & 100 \\
\hline & & & & AalbGLURla & AALF018661 & AaegGLURla & AAEL002511 & 90 \\
\hline & & & & AalbGlur5-6-7 & AALF004844 & AaegGlur5-6-7 & AAEL002518 & 100 \\
\hline & & & & Aalblr8a & AALF001584 & AaegIr8a & AAEL002922 & 89 \\
\hline & & & & Aalblr111.2 & AALF015887 & AaegIr111.2 & AAEL003249 & 66 \\
\hline & & & & Aalblr111.2-N4 & AALF002596 & AaegIr111.2 & AAEL003249 & \\
\hline & & & & Aalb|r111.2-N1 & AALF002597 & AaegIr111.2 & AAEL003249 & 65 \\
\hline & & & & Aalblr111.2-N3 & AALF002624 & AaegIr111.2 & AAEL003249 & \\
\hline & & & & Aalblr111.2-N2 & AALF002594 & AaegIr111.2 & AAEL003249 & 63 \\
\hline & & & & Aalb|r7y.1 & AALF026222 & AaegIr7y.1 & AAEL003328 & 78 \\
\hline & & & & Aalblr7k.1 & AALF011117 & AaegIr7k.1 & AAEL003340 & 78 \\
\hline & & & & Aalblr7q.2 & AALF011116 & AaegIr7q.2 & AAEL003341 & 78 \\
\hline & & & & Aalblr7q.2-N1 & AALF026221 & AaegIr7q.2 & AAEL003341 & 51 \\
\hline & & & & Aalblr7z.1 & AALF011115 & AaegIr7z.1 & AAEL003350 & 77 \\
\hline & & & & Aalblr93a & Ae2-257294 & Aaeglr93a & AAEL005012 & 89 \\
\hline & & & & Aalblr68a & AALF019550 & Aaeglr68a & AAEL005039 & 88 \\
\hline & & & & Aalb|r75| & AALF011749 & Aaeg|r75| & AAEL005875 & 89 \\
\hline & & & & Aalblr76b & AALF014911 & Aaeglr76b & AAEL006360 & 95 \\
\hline & & & & Aalblr120 & AALF009308 & AaegIr120 & AAEL007786 & 59 \\
\hline & & & & AalbNMDAR2 & AALF008566 & AaegNMDAR2 & AAEL008090 & 96 \\
\hline & & & & AalbNMDAR1 & Ae2-221592 & AaegNMDAR1 & AAEL008587 & 95 \\
\hline & & & & Aalblr75d & AALF007079 & AaegIr75d & AAEL009696 & 74 \\
\hline & & & & Aalblr25a & AALF021738 & AaegIr25a & AAEL009813 & \\
\hline & & & & Aalblr75e. 3 & AALF002385 & Aaeglr75e.3 & AAEL010775 & 7 \\
\hline & & & & Aalblr64a & AALF007249 & AaegIr64a & AAEL010880 & 87 \\
\hline & & & & Aalblr92a & AALF003583 & AaegIr92a & AAEL011001 & 75 \\
\hline & & & & Aalblr103 & AALF006904 & AaegIr103 & AAEL011122 & 59 \\
\hline & & & & Aalblr87a.2 & AALF012561 & AaegIr87a.2 & AAEL011690 & 90 \\
\hline & & & & Aalb|r87a.1 & AALF012562 & AaegIr87a.1 & AAEL011691 & 91 \\
\hline & & & & Aalblr107.1 & AALF000280 & AaegIr107.1 & AAEL011804 & 66 \\
\hline & & & & Aalblr31a & Ae2SigPSigP-240093_FR3_145-224 & AaegIr31a & AAEL013153 & 88 \\
\hline & & & & Aalbır75i & AALF014034 & AaegIr75i & AAEL013198 & 60 \\
\hline & & & & Aalblr21a & AALF023729 & AaegIr21a & AAEL013366 & 8 \\
\hline & & & & Aalbır75o & AALF008803 & AaegIr75o & AAEL014086 & 6 \\
\hline & & & & Aalblr75k.1 & AALF008804 & AaegIr75k.1 & AAEL014089 & 82 \\
\hline & & & & Aalblr40a & AALF022986 & AaegIr40a & AAEL014270 & 93 \\
\hline & & & & Aalblr40a-N1 & AALF015195 & Aaeglr40a & AAEL014270 & 77 \\
\hline & & & & Aalblr7s.1 & AALF011118 & Aaeglr7s.1 & AAEL014685 & 83 \\
\hline & & & & Aalblr7y.2 & AALF026333 & Aaeglr7y.2 & AAEL014686 & \\
\hline & & & & Aalblr7r.1 & AALF011121 & Aaeglr7r.1 & AAEL014688 & \\
\hline & & & & Aalbır100a.1 & AALF002292 & AaegIr100a.1 & AAEL015078 & \\
\hline & & & & Aalblr100e.1 & Ae2SigPSigP-173750_FR3_1-66 & AaegIr100e.1 & AAEL015079 & 95 \\
\hline & & & & Aalblr100d & AALF002291 & AaegIr100d & AAEL015265 & 83 \\
\hline & & & & Aalblr100c.2 & AALF026646 & AaegIr100c.2 & AAEL015267 & 84 \\
\hline & & & & Aalblr41-like1 & AALF009945 & AaegIr41-like1 & AAEL018060 & 75 \\
\hline & & & & Aalblr41-like2 & AALF026946 & AaegIr41-like1 & AAEL018060 & 6 \\
\hline & & & & Aalbır41-like & AALF021623 & AaegIr41-like & AAEL018359 & 5 \\
\hline
\end{tabular}

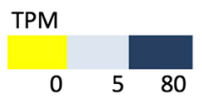

Fig. 6 (See legend on next page.) 
(See figure on previous page.)

Fig. 6 Ionotropic receptors (IR) in Ae. albopictus transcriptome. Left panel, abundance profile map: intensity scale (color gradient from blue to yellow indicates levels from high TPM to low) as indicated at the bottom. FA, female antennae; FP, female palps; MH male heads and FB, female body. IR name and Ae. albopictus ID columns indicate respectively the name assigned to IR in Ae. albopictus and the ID (VectorBase ID AALFxxxxxx when available or contig ID in the transcriptome). Ae. aegypti IR name and ID columns list the IR name in Ae. aegypti and the VectorBase code (AAELxxxxxx) of Ae. aegypti orthologs, respectively, with the percent (\%) of identity reported in the last column

a $100 \mathrm{~kb}$ region (Additional file 21: Figure S10 and Additional file 15: Dataset S5).

\section{Gustatory receptors}

We identified 30 distinct contigs encoding putative GR in the transcriptome of Ae. albopictus two of which (AalbGR20 and AalbGR63) are modestly abundant across all samples. Furthermore, as expected, three putative GRs (AalbGR1, AalbGR2 and AalbGR3) which are orthologous to a triad of GRs expressed in the maxillary palp of An. gambiae [24] and Ae. aegypti females [69] were similarly differentially abundant in the palps of $A e$. albopictus female (Fig. 3 and Fig. 7). Functional studies revealed a central role of both AaegGR1 and AaegGR3 in the perception of $\mathrm{CO}_{2}[25,26]$ which plays a crucial role in mosquito perception of both heat and the attractant lactic acid in Ae. aegypti thus supporting the hypothesis that multimodal integration of $\mathrm{CO}_{2}$, heat, and odours mediates host-seeking and blood-feeding behaviour [26]. The high degree of identity of AalbGR1 and AalbGR3 with their Ae. aegypti homologues (86\% and $91 \%$, respectively) along with data from field studies revealing that Ae. albopictus mosquitoes respond to attractants 1-octen-3-ol (octenol) and L-lactic acid [84], lead us to hypothesize that AalbGR1 and AalbGR3 are likely to be components of Ae. albopictus maxillary palp $\mathrm{CO}_{2}$ receptor complex. In addition, only a single putative GR (AalbGR58) was found to be specifically expressed and relatively abundant (TPM >3) in Ae. albopictus female antennae. Three other GRs (AalbGR20, AalbGR35 and AalbGR63) showed an ubiquitous presence in different samples of the mosquito. According to recent transcriptomic catalogues, homologues of these three receptors were not transcriptionally active in antennae and palps of Ae. aegypti mosquitoes [69, 71]. Sequence homology among these seven abundant GRs spans between 5.9 and 37\%, as reported in Additional file 22: Table S4.

\section{Other contigs overexpressed in sensory organs}

In addition to the OBP, OR, IR and GR gene families, which are often labeled as the "main chemosensory components", several contigs encoding putative proteins involved in olfaction are found among those differentially expressed in antennae and palps (FDR $<0.001$, Fig. 8). Contigs encoding for putative odorant and pheromonedegrading enzymes (ODEs and PDEs), that play essential roles in clearing sensillar lymph, were identified. Among these, members of four relevant subfamilies were found: (i) cytochrome P450 (CYPs), with 8 and 2 contigs in the antennae-and palps-specific clusters, respectively [95, 96]; (ii) esterases (ESTs) with 1 contig in the antennaeand 3 contigs in the palps-specific clusters [97, 98]; (iii) hydroxysteroid dehydrogenases (HSD), also involved in the degradation of hormones [99], with 3 and 4 contigs in the antennae- and palps-specific clusters, respectively; (iv) glutathione S-transferase (GST), which has also been implicated in odorant clearing and degradation pathways $[100,101]$ and, according to TPM values, it is one of the most abundant contigs in the antennae-enriched list. It is worth mentioning that insect GSTs are generally involved in the protection against oxidative stress, detoxification of both endogenous and xenobiotic compounds and are also entailed in intracellular transport, biosynthesis and metabolism of hormones. Moreover, a crucial role of insect GSTs in insecticide resistance was also proved [102, 103].

Beyond ODEs/PDEs other contigs were found significantly enriched in antennae and palps. One of the most abundant female antennal contig is AALF015401, which encodes a conserved secreted protein whose putative ortholog in An. gambiae (AGAP007976) is also antennaespecific and encodes a protein with as yet no function. Another antennae-enriched contig (AALF007737) encodes for a putative peptide showing similarity with antennal carrier protein AP-2 [104]. Finally, several additional antennae-enriched contigs coded for putative cuticular proteins [105], core cytoskeletal components (dynein heavy chains), juvenile hormone-inducible protein and hemolymph juvenile hormone binding protein. Some of the ODEs (esterases, cytochrome P450) as well as juvenile hormone-inducible proteins were among the most abundant contigs found in the palps. Moreover, AALF013835 that encodes for a potassium/sodium hyperpolarizationactivated cyclic nucleotide-gated channel (HCN) showed high and specific expression in Ae. albopictus palps where, in light of the odorant-gated ion channel based activity of insect chemosensory neurons, it likely plays a modulatory role in maintaining neuronal membrane potential (and therefore action potential frequency) [106]. Finally, contigs encoding for proteins possibly involved in immune defense mechanisms were found in both antennae- and palpsspecific clusters. In particular, palps-specific contigs 


\begin{tabular}{|c|c|c|c|c|c|c|c|c|}
\hline FA & FP & MH & FB & GR name & Ae. albopictus ID & GR name (Ae. aegypti) & Ae. aegypti ID & $\%$ \\
\hline & & & & AalbGr6 & AALF007327 & AaegGr6 & AAELO00012 & 87 \\
\hline & & & & AalbGr64e & AALF027206 & AaegGr64e & AAEL000043 & 88 \\
\hline & & & & AalbGr4 & AALF027205 & AaegGr4 & AAEL000048 & 94 \\
\hline & & & & AalbGr7 & AALF007326 & AaegGr7 & AAEL000060 & 82 \\
\hline & & & & AalbGr9 & AALF003503 & AaegGr9 & AAEL000075 & 90 \\
\hline & & & & AalbGr10 & AALF024475 & AaegGr10 & AAEL000082 & 87 \\
\hline & & & & AalbGr34 & Ae2-100900 & AaegGr34 & AAEL000162 & 97 \\
\hline & & & & AalbGr2 & AALF019900 & AaegGr2 & AAEL002167 & 96 \\
\hline & & & & AalbGr1 & AALF013834 & AaegGr1 & AAEL002380 & 86 \\
\hline & & & & AalbGr45 & AALF018950 & AaegGr45 & AAEL006494 & 68 \\
\hline & & & & AalbGr41 & AALF018954 & AaegGr41 & AAEL006500 & 59 \\
\hline & & & & AalbGr64 & AALF016270 & AaegGr64 & AAEL007142 & 75 \\
\hline & & & & AalbGr20 & AALF009050 & AaegGr20 & AAEL007935 & 96 \\
\hline & & & & AalbGr76 & AALF028583 & AaegGr76 & AAEL009545 & 80 \\
\hline & & & & AalbGr3 & AALF019265 & AaegGr3 & AAEL010058 & 91 \\
\hline & & & & AalbGr53 & AALF008393 & AaegGr53 & AAEL010279 & 74 \\
\hline & & & & AalbGr73 & AALF023741 & AaegGr73 & AAEL010962 & 91 \\
\hline & & & & AalbGr11 & AALF026442 & AaegGr11 & AAEL011174 & 82 \\
\hline & & & & AalbGr14 & AALF004249 & AaegGr14 & AAEL011571 & 94 \\
\hline & & & & AalbGr25 & AALF019938 & AaegGr25 & AAEL013193 & 65 \\
\hline & & & & AalbGr26 & Ae2-30210 & AaegGr26 & AAEL013195 & 66 \\
\hline & & & & AalbGr23 & AALF019937 & AaegGr23 & AAEL013196 & 79 \\
\hline & & & & AalbGr46 & AALF018952 & AaegGr46 & AAEL017092 & 74 \\
\hline & & & & AalbGr61 & AALF009051 & AaegGr61 & AAEL017202 & 55 \\
\hline & & & & AalbGr61-N1 & AALF025226 & AaegGr61 & AAEL017202 & 63 \\
\hline & & & & AalbGr71 & AALF017431 & AaegGr71 & AAEL017307 & 69 \\
\hline & & & & AalbGr78 & AALF025209 & AaegGr78 & AAEL017312 & 82 \\
\hline & & & & AalbGr58 & Ae2SigPSigP-643_FR3_1-225 & AaegGr58 & AAEL017324 & 73 \\
\hline & & & & AalbGr35 & Ae2SigPSigP-91626_FR6_1-238 & AaegGr35 & AAEL017415 & 88 \\
\hline & & & & AalbGr63 & AALF021200 & AaegGr63 & AAEL017565 & \\
\hline
\end{tabular}

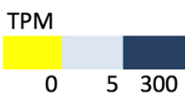

Fig. 7 Gustatory receptors (GR) in Ae. albopictus transcriptome. Left panel, abundance profile map: intensity scale (color gradient from blue to yellow indicates levels from high TPM to low) as indicated at the bottom. FA, female antennae; FP, female palps; MH male heads and FB, female body. GR name and Ae. albopictus ID columns indicate respectively the name assigned to GR in Ae. albopictus and the ID (VectorBase ID AALFxxxxxx when available or contig ID in the transcriptome). Ae. aegypti GR name and ID columns list the GR name in Ae. aegypti and the VectorBase code (AAELxxxxxx) of Ae. aegypti orthologs, respectively, with the percent (\%) of identity reported in the last column

encoding for leucine rich repeats protein (LRR, AALF017691), prophenoloxidase (PPO, AALF012718), cecropin C (CEC, Ae2SigPSigP-213035_FR1_18-90), and for a member of the nimrod family (AALF007372) were identified.

\section{Genes involved in other sensory modalities}

In addition to the sensory genes described so far, several other protein families play crucial roles in insect sensing of the external environment. These proteins are involved in different physiological pathways such as mechanosensation, thermosensation, perception of taste, light, humidity and gravity, sound detection and other forms of stimuli. A selection of these sensory genes and their abundance profiles is shown in Fig. 8, that highlights also their wide functional overlap, with members of a gene family often involved in more than one perception pathway (see ppk family members taking part to both mechano- and taste/chemo-perception). We will describe some of the most relevant protein families involved in i) taste- and chemo-perception, ii) mechanosensation and iii) thermosensation.

\section{Chemo- and taste-perception}

Members of the CheA/CheB gene family are small and secreted proteins mainly involved in perception of chemical stimuli and most of them interact with ppk channels. Using a list of 10 Ae. aegypti CheA and 3 CheB sequences $[69,79]$ we have identified 17 Ae. albopictus contigs. Of these, only 3 CheA/B transcripts (AALF012531, AALF021726 and Ae2-204624_FR6_8206) display TPM $>1$ in antennae and/or palps. In particular, AALF021726 (orthologs of AAEL012061) is by far the most abundant (TPM > 10) and specific CheA of female antennae, differently from its Ae. aegypti ortholog $(\mathrm{TPM}<1$ in the antennae, [71]). Ae2204624_FR6_8-206 (ortholog of AAEL009978, very scarce in Ae. aegypti antennae, TPM $<1$ ) is abundant in female antennae $(\mathrm{TPM}=3.6)$ and to a lesser extent in the palps $(\mathrm{TPM}=1.0$ ) (Fig. 8 and Additional file 23: Table S5). Both CheA/CheB are highly expressed in the 


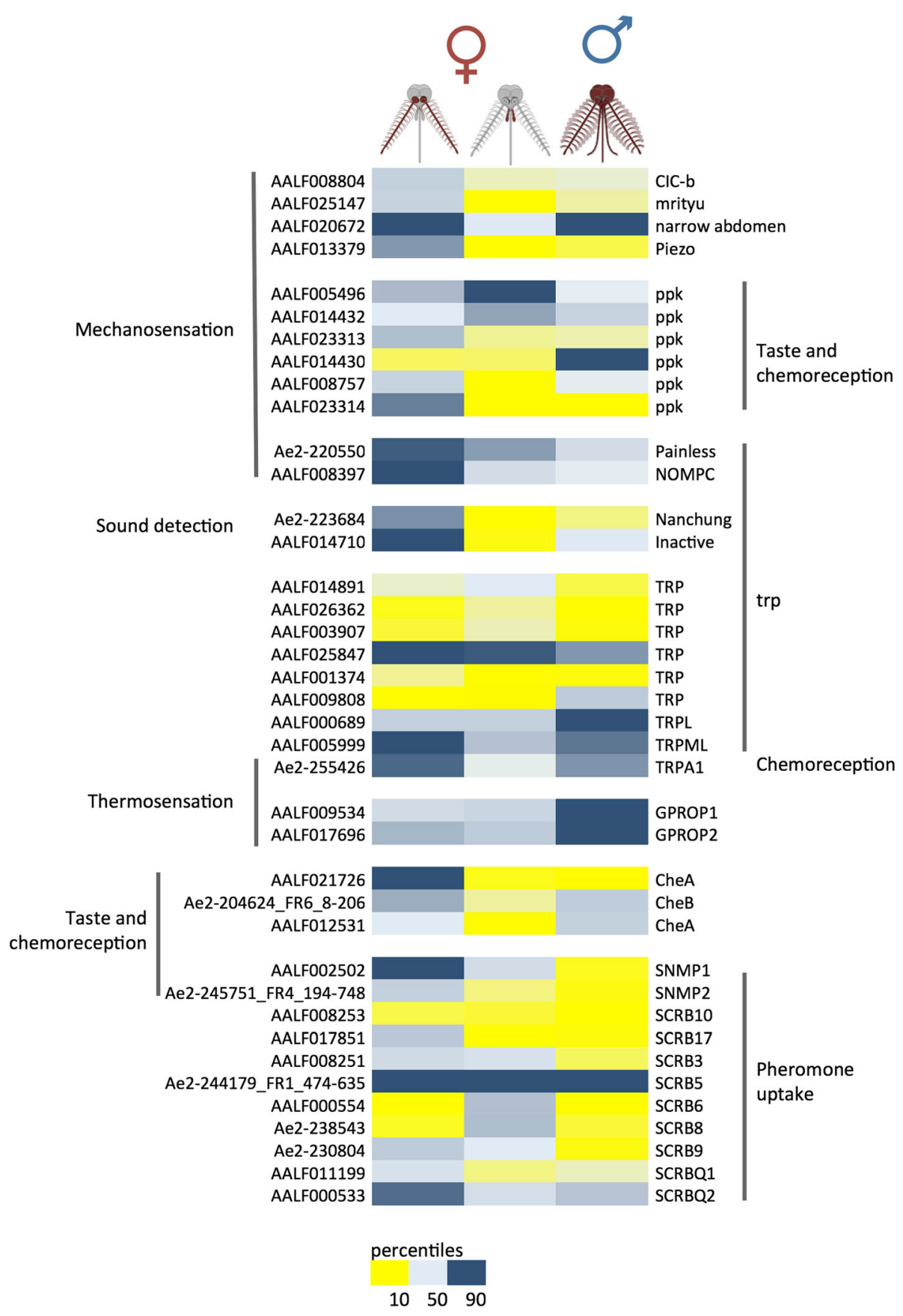

Fig. 8 Abundance profiles of sensory genes in Ae. albopictus. Abundance profile map: intensity scale (color gradient from blue to yellow indicates levels from high TPM to low) as indicated at the bottom. Gene name and contig ID (VectorBase codes when available) are also reported

tarsi of Ae. aegypti mosquitoes [23] and at a lesser extent in maxillary palps [69]. Moreover, orthologs of AALF021726 and Ae2-204624_FR6_8-206 in Ae. aegypti mosquitoes are the most abundant members of CheA and CheB expressed in the labella [23]. The third member of Ae. albopictus CheA family (AALF012531), putative ortholog of AAEL004928, was found expressed in female antennae and male heads.
Members of the pickpocket gene family (degenerin/epithelial $\mathrm{Na}^{+}$channel, DEG/ENaC) and members of the trp family (transient receptor potential channels) are associated with taste perception, photo-, thermo-, and mechanoreception [46]. In mosquitoes, maxillary palps and proboscis are head appendages that along with the tarsi and labella play an essential role in gustatory pathways $[23,69,79,107]$. We have identified $18 \mathrm{ppk}$ 
receptors in the Ae. albopictus transcriptome (Additional file 23: Table S5). Of these, 5 contigs have a TPM value $>1$ (Additional file 23: Table S5) in at least one sensory appendage library, with two abundance patterns: i) contigs enhanced in antennae vs palps (AALF023313, AALF023314 and AALF008757); ii) contigs that are more abundant in female maxillary palps than antennae (AALF005496 and AALF014432).

SNMPs belong to the scavenger receptor type $B$ gene family (SCRB/CD36) [32, 33], are involved in a more wide range of functions. Using 13 Ae aegypti putative SCRB genes as query we identified 12 putative orthologs (identity $>73 \%$ ) in Ae. albopictus. SNMP1 was strongly enhanced in female antennae, while SNMP2 showed a more ubiquitous expression profile (Additional file 23: Table S5 and Fig. 8) as also reported in Ae. aegypti [79]. Among the 11 other SCRBs found in Ae. aegypti sensory organs [69], we identified 10 orthologs in Ae. Albopictus. Overall, they are abundantly expressed in head chemosensory appendages, with SCRB5 and SCRB6 highly enriched in maxillary palps $(\mathrm{TPM}=923$ and $\mathrm{TPM}=40$, respectively), similarly to Ae. aegypti [71]. The exact role of these proteins in head organs is unknown, however, they are well conserved among most insect species and may also have non-chemosensory roles [34].

\section{Thermosensation}

Several members of the TRP gene family, composed by 13 genes belonging to 7 subfamilies, are involved in temperature perception and other aspects of insect behaviour, including avoidance of noxious heat/odorants/ tastants [46]. Noxious heat perception is most likely carried out by antennae as reported in Ae. aegypti [108] and An. gambiae [48]. TRPA1 is a heat-activated channel expressed in thermosensitive sensilla of An. gambiae female antennae [48]. We have identified TRPA1 ortholog in Ae. albopictus and several contigs with high level of similarity to other Ae. aegypti TRP family members. Painless (another TRPA family member) also associated with avoidance of dry climates and mechanosensory stimuli was shown to be expressed in Ae. albopictus antennae and palps. Finally, we have identified the putative homolog of Ae. aegypti TRPA family gene pyrexia in Ae. albopictus transcriptome albeit with low level of abundance in sensory organs.

Members of rhodopsin family of GPCRs (G proteincoupled receptors) are known to play a central role in photoreception (vision) [6], however, they may be also involved in the perception of temperature, since an overlap between genes involved in light detection and in temperature discrimination is known in insects [109]. Most of the Ae. albopictus GPROP family members are highly abundant in male heads, as expected with their main role in vision. GPROP1 and GPROP2 are also highly abundant in female antennae and palps (Additional file 23: Table S5), whereas only basal levels were observed for other members of the family (GPROP3, GPROP5, GPROP8 and GPROP9). The arrestin family is a small group of genes involved in the termination and desensitization of GPCR-based transduction as well as a range of downstream cellular signalling pathways [110]. We identified Ae. albopictus arrestin1 and arrestin2 genes, that, as expected, were overexpressed in male heads, which likely reflects their role in the ommatidia of insects where they have been shown to quench the photo response by directly interacting with photoactivated rhodopsin GPCRs [111, 112]. Arrestin1 and arrestin2 have also been characterized in olfactory tissues of both Drosophila and An. gambiae [113], and are not surprisingly detected also in the maxillary palps of $A e$. aegypti mosquitoes [69], providing a rationale for our detection of the Ae. albopictus ortholog for arrestin1 expression in female palps and antennae.

\section{Mechanosensation}

Transduction of mechanical stimuli plays an essential role in many aspects of mosquito life cycle that include hearing, touch and pain sensitivity [6]. Ae. albopictus piezo, an evolutionarily conserved transmembrane protein involved in mechanosensory transduction in Drosophila [51] is relatively abundant in antennae (Fig. 8 and Additional file 23: Table S5). In Ae. albopictus, transcripts encoding for both piezo and the TRP channel painless are indeed abundant not only in antennae but also in female bodies, suggesting that they are also part of sensory neurons located in mosquito antennae. Other genes that have been implicated in mild touch detection appear to be specific both of female antennae, such as $n o m p C$, and of male sensory appendages of the head, such as Nmdarl. In our study, Narrow abdomen appears enhanced presumably in the antennae of both sexes, while Cicb is ubiquitous. Sound detection in D. melanogaster requires the TRPV channels encoded by Nanchung (Nan), inactive (Iav) and nompC [114] that are abundantly expressed in many head appendages [46]. Similarly to abundance profiles in Ae. aegypti $[69,71]$, we observed robust abundance of these genes in $A e$. albopictus female antennae but not in maxillary palps, a pattern only partially overlapping with data from $A n$. gambiae, where Nanchung and inactive are believed to play a role in the capacity of male antennae to detect female wing beats during mating swarms [83, 115].

\section{qPCR validation}

To validate the transcriptome profiles obtained by RNAseq analysis, we have selected 11 genes belonging to the four main chemosensory families for analysis by quantitative real time PCR. The study was performed using independent, duplicate RNA samples extracted from 

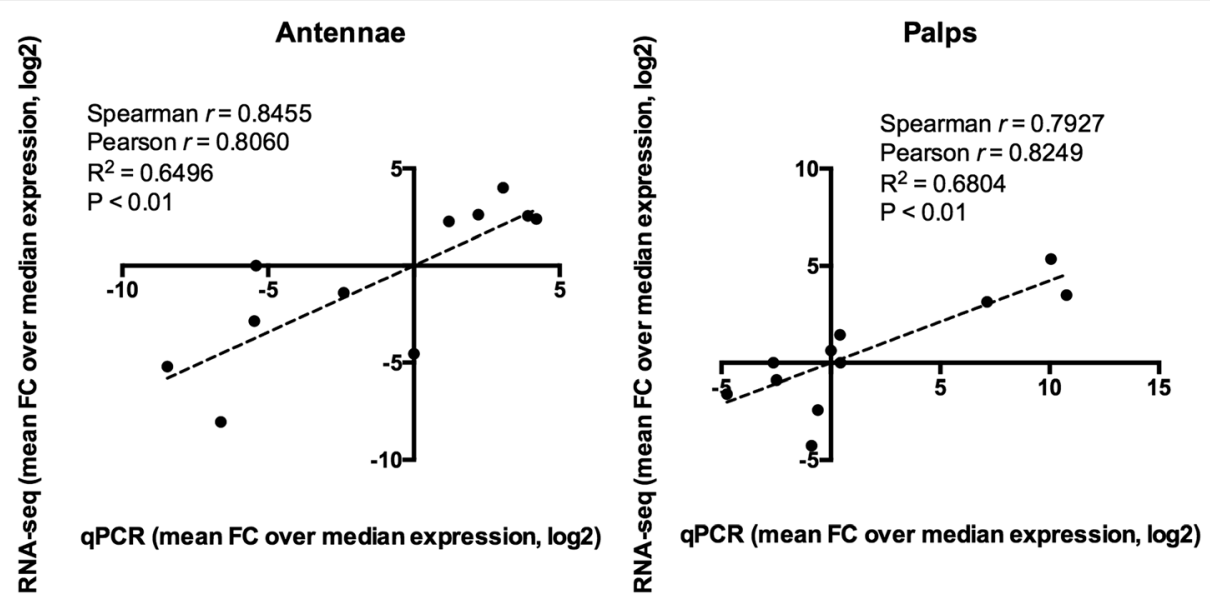

Fig. 9 qPCR validation. Correlation between transcriptional abundance of 11 genes in both antennae (a) and maxillary palps (b) revealed by qPCR and RNA-seq. Level of abundance is defined as the ratio between each sample value over the group median (fold change, FC) in both qPCR and RNA-seq approaches. For both techniques, statistical evaluation throughout Spearman and Pearson tests was performed and results are reported in the figure insets

female antennae and maxillary palps. Targets were chosen so as to include those with high, medium or low abundance levels in the different tissues based on our collective RNA-seq analysis. Four OBPs (AalbOBP36, AalbOBP47 and AalbOBP83, all of them highly enriched in the antennae, and AalbOBP27, expressed at the same high level - TPM $>500$ - in both tissues), two ORs (AalbOR8 and AalbOR84, showing a slight enhancement of expression in palps and antennae, respectively), the ubiquitously abundant ORCO, two GRs (the palps-specific AalbGR3 and AalbGR58, that shows a weak - TPM $<10$ - but specific expression in the antennae) and finally, two ubiquitous IRs, the abundant AalbIR25a and the weakly expressed AalbNMDAR1. Correlation studies indicate statistically significant $(P<0.01)$ linear relationships between datasets obtained from the two methods for both antennae and palps samples (Fig. 9). Several parameters might influence both methods, determining discrepancies and variability among abundance profiles. Even though the correlation between the two techniques is strong, a few differences can be highlighted and could provide valuable new information. In particular, it is worth mentioning AalbGR58 expression in antennae, detected at low level by RNA-seq $(\mathrm{TPM}<10)$ while revealed abundant and specific by qPCR (Additional file 3: Figure S11).

\section{Conclusions}

We present the RNA-seq generated transcriptome profiles of the main sensory appendages of the tiger mosquito Ae. albopictus. This study provides a tissuespecific survey of transcript abundance in adult Ae. albopictus sensory organs, providing the community with a first catalogue of annotated chemosensory genes. We have analyzed antennae and maxillary palps of female mosquitoes, male heads and whole female bodies as reference samples for differential expression studies. Around 350 putative proteins were classified in several functional families involved in mosquito olfaction and more generally in the perception of the surrounding environment. Our analysis also provided an important contribution for the improvement of genome annotation since most of the transcripts/proteins were manually annotated to describe molecular features and gene clusters were verified on released genomic scaffolds. In addition, Ae. albopictus chemosensory genes belonging to OBP, OR, IR and GR gene families were compared to homologous groups of genes identified in other mosquito species such as the malaria mosquito An. gambiae, the southern house mosquito $C$. quinquefasciatus and, in particular, the dengue and yellow fever mosquito Ae. aegypti. The comparison allowed identifying bona fide orthologs, pointing out differences in sequence and gene abundance as well as highlighting cases of gene gain/ loss. Indeed, variation in abundance of olfactory/sensory genes between Ae. aegypti and Ae. albopictus may provide a first starting point to study and better understand physiological and behavioral differences between these two mosquito species.

\section{Additional files}

Additional file 1: Alignment files. MEGA files of protein alignments and tree. (ZIP 93 kb)

Additional file 2: Table S6. Features of primers used in this study. (PDF $44 \mathrm{~kb}$ )

Additional file 3: Figure S11. Correlation of biological replicates. (PDF 951 kb)

Additional file 4: Dataset S1. Transcriptome: sequence features of contigs. (XLSX 12364 kb)

Additional file 5: Dataset S2. Transcriptome: comparisons with databases. (XLSX 15138 kb) 
Additional file 6: Dataset S3. Transcriptome: differential expression analysis. (XLSX $14649 \mathrm{~kb}$ )

Additional file 7: Dataset S4. Transcriptome: sequences. (XLSX 16036 kb) Additional file 8: Figure S1. Differential expression, cluster analysis and sample correlation matrix. (PDF $499 \mathrm{~kb}$ )

Additional file 9: Figure S2. GO terms enrichment. (pdf) (PDF 385 kb)

Additional file 10: Table S1. Chemosensory proteins in the different samples and average TPM. (PDF $41 \mathrm{~kb}$ )

Additional file 11: Figure S3. Alignment of Classic OBPs.(PDF $7646 \mathrm{~kb})$ Additional file 12: Figure S4. Alignment of PlusC OBPs. (PDF 4328 kb) Additional file 13: Figure S5. Alignment of Atypical OBPs. (PDF $8211 \mathrm{~kb}$ ) Additional file 14: Figure S6. Phylogram of OBP family members in $A e$. albopictus and Ae. aegypti. (PDF $125 \mathrm{~kb}$ )

Additional file 15: Dataset S5. Mapping of chemosensory genes on Ae. albopictus genomic scaffolds. (XLSX $79 \mathrm{~kb}$ )

Additional file 16: Table S2. Comparison with previously published A. albopictus OBP. (XLSX $14 \mathrm{~kb}$ )

Additional file 17: Table S3. Comparison with published $\boldsymbol{A}$. albopictus OBP and OR in Chen X-G et al. (2015) [48]. (XLSX 67 kb)

Additional file 18: Figure S7. Alignment and phylogenetic tree of OR61. (PDF $1577 \mathrm{~kb}$ )

Additional file 19: Figure S8. Alignment and phylogenetic tree of IR41p1. (PDF $1841 \mathrm{~kb}$ )

Additional file 20: Figure S9. IR111.2 gene family in Ae. albopictus. (PDF $144 \mathrm{~kb}$ )

Additional file 21: Figure S10. IR7 genetic cluster. (PDF 250 kb)

Additional file 22: Table S4. Percentage of identity of gustatory receptors in Ae. albopictus sensory organs. (PDF $43 \mathrm{~kb}$ )

Additional file 23: Table S5. Sensory genes in Ae. albopictus transcriptome. (XLSX 52 kb)

\section{Abbreviations}

CDS: Coding sequences; CEC: Cecropin C; CYP: Cytochrome P450; DEG/ ENaC: Degenerin/epithelial Na + channel; EST: Esterases; FA: Female antennae; FB: Female whole body; FC: Fold change; FDR: False discovery rates; FP: Female palps; GO: Gene ontology; GPCR: G protein-coupled receptor; GR: Gustatory receptors; GRN: Gustatory receptor neuron; GST: Glutathione S-transferase; HCN: Potassium/sodium hyperpolarizationactivated cyclic nucleotide-gated hannel; HSD: Hydroxysteroid dehydrogenases; lav: Inactive; iGluRs: Ionotropic glutamate receptors; IR: Ionotropic receptors; JHBP: Haemolymph juvenile hormone binding protein; LRR: Leucine rich repeats protein; MA plot: plot using an M (log ratios) and $\mathrm{A}$ (mean average) scale; $\mathrm{MH}$ : Male heads; Nan: Nanchung; Nmdar: N-methyl-D-aspartate receptor; nompC: No Mechanoreceptor potential C; OBP: Odorant binding protein; ODE/PDE: Odorant/pheromonedegrading enzyme; OR: Odorant receptor; ORF: Open reading frame; ORN: Olfactory receptor neuron; PBP: Pheromone binding protein; PCA: Principal component analysis; PFAM: Protein family; PlcB: Phospholipase C; ppk: Pickpocket; PPO: Prophenoloxidase; qPCR: Quantitative polymerase chain reaction; SCRB/CD36: Scavenger receptor type B/CD36; SNMP: Sensory Neuron membrane protein; TPM: Transcripts per kilobase per million; TRP: Transient receptor potential channel; TSA: Transcriptome shotgun assembly

\section{Acknowledgements}

We thank Paola Serini for mosquito rearing and Agnese Maierini for technical help.

\section{Funding}

This work was supported in the framework of the European network INFRAVEC (2011) by funding FL for the project: "Defining the olfactory repertoire of the tiger mosquito Aedes albopictus" (Integrating Activity number 228421). The work was also supported by Sapienza University of Rome, thanks to a Research Project (2014) funded to BA (C26A14AKKH). BA was also supported by funds from the European Union grant INFRAVEC (228421). JMR was supported by the intramural program of the National Institute of Allergy and Infectious Diseases. Because JMR is a government employee and this is a government work, the work is in the public domain in the United States. Notwithstanding any other agreements, the NIH reserves the right to provide the work to PubMedCentral for display and use by the public, and PubMedCentral may tag or modify the work consistent with its customary practices. You can establish rights outside of the U.S. subject to a government use license.

\section{Availability of data and materials}

Data from Illumina deep-sequencing were deposited to the NCBI Transcriptome Shotgun Assembly (TSA) Sequence Database as BioProject Accession Number PRJNA314492. Gene set annotations and expression data are available (Additional file 4: Dataset S1, Additional file 5: Dataset S2, Additional file 6: Dataset S3) with this manuscript. Sequences are also provided (Additional file 7: Dataset S4).

\section{Authors' contributions}

Conceived the project: FL and BA. Conducted the experiments: FL, TN and CF. Analysed the results: FL, MS, JMR and BA. Performed bioinformatics analyses: MS and JMR. Wrote the first draft of the manuscript and figures: FL. Revised and edited the manuscript: FL, BA, JMR, TN, MS and LJZ. All authors read and approved the final manuscript.

\section{Ethics approval and consent to participate}

Not applicable

\section{Consent for publication}

Not applicable

\section{Competing interests}

The authors declare that they have no competing interests.

\section{Publisher's Note}

Springer Nature remains neutral with regard to jurisdictional claims in published maps and institutional affiliations.

\section{Author details}

${ }^{1}$ Department of Public Health and Infectious Diseases, Division of Parasitology, Sapienza University of Rome, Rome, Italy. ${ }^{2}$ Department of Biology, University of Naples Federico II, Naples, Italy. ${ }^{3}$ Department of Life Sciences, Imperial College London, London, UK. ${ }^{4}$ Department of Biological Sciences, Vanderbilt University, Nashville, TN, USA. ${ }^{5}$ NIAID, Laboratory of Malaria and Vector Research, NIH, Rockville 20852, MD, USA.

Received: 3 July 2017 Accepted: 2 October 2017

Published online: 11 October 2017

\section{References}

1. Bonizzoni M, Gasperi G, Chen X, James AA. The invasive mosquito species Aedes Albopictus: current knowledge and future perspectives. Trends Parasitol. 2013;29:460-8.

2. Paupy C, Delatte H, Bagny L, Corbel V, Fontenille D. Aedes Albopictus, an arbovirus vector: from the darkness to the light. Microbes Infect. 2009;11:1177-85.

3. Lessler J, Chaisson LH, Kucirka LM, Bi Q, Grantz K, Salje H, et al. Assessing the global threat from Zika virus. Science. 2016;353:aaf8160.

4. Schaffner F, Mathis A. Dengue and dengue vectors in the WHO European region: past, present, and scenarios for the future. Lancet Infect Dis. 2014;14: 1271-80.

5. Medlock JM, Hansford KM, Schaffner F, Versteirt V, Hendrickx G, Zeller H, et al. A review of the invasive mosquitoes in Europe: ecology, public health risks, and control options. Vector-Borne Zoonotic Dis. 2012;12:435-47.

6. Montell C, Zwiebel LJ. Chapter Ten - Mosquito Sensory Systems. Adv In Insect Phys. 2016;51:293-328.

7. Takken W, Verhulst NO. Host preferences of blood-feeding mosquitoes. Annu Rev Entomol. 2013;58:433-53.

8. Zwiebel $L$, Takken W. Olfactory regulation of mosquito-host interactions. Insect Biochem Mol Biol. 2004;34:645-52.

9. Carey AF, Carlson JR. Insect olfaction from model systems to disease control. Proc Natl Acad Sci U S A. 2011;108:12987-95.

10. Rützler M, Zwiebel LJ. Molecular biology of insect olfaction:recent progress and conceptual models. J Comp Physiol A Neuroethol. Sens Neural Behav Physiol. 2005;191:777-90. 
11. Hallem EA, Dahanukar A, Carlson JR. Insect odor and taste receptors. Annu Rev Entomol. 2006;51:113-35.

12. Leal WS. Odorant reception in insects: roles of receptors, binding proteins, and degrading enzymes. Annu Rev Entomol. 2013;58: 120928130709004.

13. Pitts RJ, Zwiebel LJ. Antennal sensilla of two female anopheline sibling species with differing host ranges. Malar J. 2006/04/01. 2006;5:26.

14. Sato K, Pellegrino M, Nakagawa T, Nakagawa T, Vosshall LB, Touhara K. Insect olfactory receptors are heteromeric ligand-gated ion channels. Nature. 2008;452:1002-6.

15. Wicher D, Schafer R, Bauernfeind R, Stensmyr MC, Heller R, Heinemann $\mathrm{SH}$, et al. Drosophila odorant receptors are both ligand-gated and cyclic-nucleotide-activated cation channels. Nature. 2008;452:1007-U10.

16. Stengl M, Funk NW. The role of the coreceptor Orco in insect olfactory transduction. J Comp Physiol A Neuroethol Sens Neural Behav Physiol. 2013;199:897-909.

17. Bohbot JD, Pitts RJ. The narrowing olfactory landscape of insect odorant receptors. Front Ecol Evol. 2015;3:1-10.

18. Carey AF, Wang G, Su C-Y, Zwiebel LJ, Carlson JR. Odorant reception in the malaria mosquito Anopheles Gambiae. Nature. 2010;464:66-71.

19. Mcbride CS, Baier F, Omondi AB, Spitzer SA, Lutomiah J, Sang R, et al. Evolution of mosquito preference for humans linked to an odorant receptor. Nature. 2014;515:222-7.

20. Scott K, Brady R, Cravchik A, Morozov P, Rzhetsky A, Zuker C, et al. A chemosensory gene family encoding candidate gustatory and olfactory receptors in drosophila. Cell. 2001;104:661-73.

21. Wang Z, Singhvi A, Kong P, Scott K. Taste representations in the drosophila brain. Cell. 2004;117:981-91.

22. Kent LB, Walden KKO, Robertson HM. The Gr family of candidate gustatory and olfactory receptors in the yellow-fever mosquito Aedes Aegypti. Chem Senses. 2008;33:79-93.

23. Sparks JT, Vinyard BT, Dickens JC. Gustatory receptor expression in the labella and tarsi of Aedes Aegypti. Insect Biochem Mol Biol. 2013:43:1161-71.

24. Lu T, Qiu YT, Wang G, Kwon JY, Rutzler M, Kwon HW, et al. Odor coding in the maxillary palp of the malaria vector mosquito Anopheles Gambiae. Curr Biol. 2007:17:1533-44

25. Erdelyan CNG, Mahood TH, Bader TSY, Whyard S. Functional validation of the carbon dioxide receptor genes in Aedes Aegypti mosquitoes using RNA interference. Insect Mol Biol. 2012;21:119-27.

26. McMeniman CJ, Corfas RA, Matthews BJ, Ritchie SA, Vosshall LB. Multimodal integration of carbon dioxide and other sensory cues drives mosquito attraction to humans. Cell. 2014;156:1060-71.

27. Croset V, Rytz R, Cummins SF, Budd A, Brawand D, Kaessmann H, et al. Ancient protostome origin of chemosensory ionotropic glutamate receptors and the evolution of insect taste and olfaction. PLoS Genet. 2010;6

28. Rytz R, Croset V, Benton R. lonotropic receptors (IRs): chemosensory ionotropic glutamate receptors in drosophila and beyond. Insect Biochem Mol Biol. 2013;43:888-97.

29. Pitts RJ, Derryberry SL, Zhang Z, Zwiebel LJ. Variant lonotropic receptors in the malaria vector mosquito Anopheles Gambiae tuned to amines and carboxylic acids. Sci Rep. 2017;7:40297.

30. Silbering AF, Rytz R, Grosjean Y, Abuin L, Ramdya P, GSXE J, et al. Complementary function and integrated wiring of the evolutionarily distinct drosophila olfactory subsystems. J Neurosci. 2011;31:13357-75.

31. Hussain A, Zhang M, Üçpunar HK, Svensson T, Quillery E, Gompel N, et al. Ionotropic chemosensory receptors mediate the taste and smell of polyamines. PLoS Biol. 2016;14:1-30.

32. Rogers $M E$, Sun $M$, Lerner MR, Vogt RG. Snmp-1, a novel membrane protein of olfactory neurons of the silk moth Antheraea Polyphemus with homology to the CD36 family of membrane proteins. J Biol Chem. 1997;272:14792-9.

33. Rogers ME, Krieger J, Vogt RG. Antennal SNMPS (sensory neuron membrane proteins) of lepidoptera define a unique family of invertebrate CD36-like proteins. J Neurobiol. 2001;49:47-61.

34. Vogt RG, Miller NE, Litvack R, Fandino RA, Sparks J, Staples J, et al. The insect SNMP gene family. Insect Biochem Mol Biol. 2009;39:448-56.

35. Benton R, Vannice KS, Vosshall LB. An essential role for a CD36-related receptor in pheromone detection in drosophila. Nature. 2007;450:289-93.
36. Jin $\mathrm{X}, \mathrm{Ha}$ TS, Smith DP. SNMP is a signaling component required for pheromone sensitivity in drosophila. Proc Natl Acad Sci U S A. 2008;105:10996-1001.

37. Vieira FG, Rozas J. Comparative genomics of the odorant-binding and chemosensory protein gene families across the arthropoda: origin and evolutionary history of the chemosensory system. Genome Biol Evol. 2011;3:476-90.

38. Manoharan M, Ng Fuk Chong M, Vaïtinadapoulé A, Frumence $E$, Sowdhamini R, Offmann B. Comparative genomics of odorant binding proteins in Anopheles Gambiae, Aedes Aegypti, and Culex Quinquefasciatus. Genome Biol Evol. 2013;5:163-80.

39. Murphy EJ, Booth JC, Davrazou F, Port AM, Jones DNM. Interactions of anopheles gambiae odorant-binding proteins with a human-derived repellent: implications for the mode of action of N,N-diethyl-3methylbenzamide (DEET). J Biol Chem. 2013;288:4475-85.

40. Lagarde A, Spinelli S, Tegoni M, He X, Field L, Zhou JJ, et al. The crystal structure of odorant binding protein 7 from Anopheles Gambiae exhibits an outstanding adaptability of its binding site. J Mol Biol. 2011;414:401-12.

41. Biessmann $H$, Andronopoulou E, Biessmann MR, Douris V, Dimitratos SD, Eliopoulos E, et al. The Anopheles Gambiae odorant binding protein 1 (AgamOBP1) mediates indole recognition in the antennae of female mosquitoes. PLoS One. 2010;5:e9471.

42. Millar JG, Chaney JD, Mulla MS. Identification of oviposition attractants for Culex Quinquefasciatus from fermented Bermuda grass infusions. J Am Mosq Control Assoc U S. 1992:8:11-7.

43. Ben-Shahar Y, Lu B, Collier DM, Snyder PM, Schnizler M, Welsh MJ. The drosophila gene CheB42a is a novel modifier of deg/ENaC channel function. PLoS One. 2010;5:e9395.

44. Park SK, Mann KJ, Lin H, Starostina E, Kolski-Andreaco A, Pikielny CW. A drosophila protein specific to pheromone-sensing gustatory hairs delays males' copulation attempts. Curr Biol. 2006;16:1154-9.

45. Suh E, Bohbot JD, Zwiebel LJ. Peripheral olfactory signaling in insects. Curr Opin Insect Sci. 2014;6

46. Fowler MA, Montell C. Drosophila TRP channels and animal behavior. Life Sci. 2013;92:394-403.

47. Ni L, Bronk P, Chang EC, Lowell AM, Flam JO, Panzano VC, et al. A gustatory receptor paralogue controls rapid warmth avoidance in drosophila. Nature. 2013;500:580-4.

48. Wang G, Qiu YT, Lu T, Kwon HW, Pitts RJ, Van Loon JJ, et al. Anopheles Gambiae TRPA1 is a heat-activated channel expressed in thermosensitive sensilla of female antennae. Eur J Neurosci. 2009;30:967-74.

49. Corfas RA, Vosshall LB. The cation channel TRPA1 tunes mosquito thermotaxis to host temperatures. elife. 2015;4:1-16.

50. Tracey WD, Wilson Rl, Laurent G, Benzer S. Painless, a drosophila gene essential for Nociception. Cell. 2003;113:261-73.

51. Kim SE, Coste B, Chadha A, Cook B, Patapoutian A. The role of drosophila Piezo in mechanical nociception. Nature. 2012;483:209-12.

52. Johnson W A., carder JW. Drosophila Nociceptors mediate larval aversion to dry surface environments utilizing both the painless TRP Channel and the DEG/ENaC subunit, PPK1. PLoS One 2012;7:e32878.

53. Walker RG, Willingham AT, Zuker CS. A drosophila mechanosensory transduction channel. Science. 2000;287:2229-34.

54. Yan Z, Zhang W, He Y, Gorczyca D, Xiang Y, Cheng LE, et al. Drosophila NOMPC is a mechanotransduction channel subunit for gentle-touch sensation. Nature. 2012;493:221-5.

55. Rezza G, Nicoletti L, Angelini R, Romi R, Finarelli AC, Panning M, et al. Infection with chikungunya virus in Italy: an outbreak in a temperate region. Lancet. 2007;370:1840-6.

56. Gould EA, Gallian P, De Lamballerie X, Charrel RN. First cases of autochthonous dengue fever and chikungunya fever in France: from bad dream to reality! Clin Microbiol Infect. 2010;16:1702-4.

57. Grandadam M, Caro V, Plumet $S$, Thiberge $J M$, Souarès $Y$, Failloux $A B$, et al. Chikungunya virus, southeastern France. Emerg Infect Dis. 2011;17:910-3.

58. La Ruche $G$, Souarès $Y$, Armengaud A, Peloux-Petiot F, Delaunay $P$, Desprès $P$, et al. First two autochthonous dengue virus infections in metropolitan France, September 2010. Euro Surveill Bull. 2010;15:19676.

59. Gjenero-Margan I, Aleraj B, Krajcar D, Lesnikar V, Klobucar A, Pem-Novosel I, et al. Autochthonous dengue fever in Croatia, august- September 2010. Eur Secur. 2011;16:1-4

60. Wilder-Smith A, Quam M, Sessions O, Rocklov J, Liu-Helmersson J, Franco L, et al. The 2012 dengue outbreak in Madeira: exploring the origins. Erratum appears in Euro Surveill. 2014;19(9):20725]. Euro Surveill. Bull. 2014;19:20718. 
61. Dritsou V, Topalis P, Windbichler N, Simoni A, Hall A, Lawson D, et al. A draft genome sequence of an invasive mosquito: an Italian Aedes albopictus. Pathog Glob Health. 2015;109:207-20.

62. Chen X-G, Jiang X, Gu J, Xu M, Wu Y, Deng Y, et al. Genome sequence of the Asian Tiger mosquito, Aedes Albopictus, reveals insights into its biology, genetics, and evolution. Proc Natl Acad Sci U S A. 2015;112:E5907-15.

63. Foster WA, Takken W. Nectar-related vs. human-related volatiles: behavioural response and choice by female and male Anopheles Gambiae (Diptera: Culicidae) between emergence and first feeding. Bull Entomol Res. 2004;94:145-57.

64. Omondi BA, Majeed S, Ignell R. Functional development of carbon dioxide detection in the maxillary palp of Anopheles Gambiae. J Exp Biol. 2015;218:2482-8.

65. Bohbot JD, Durand NF, Vinyard BT, Dickens JC. Functional development of the octenol response in Aedes Aegypti. Front Physiol. 2013;4 MAR:1-8.

66. Musacchia F, Basu S, Petrosino G, Salvemini M, Sanges R. Annocript: a flexible pipeline for the annotation of transcriptomes also able to identify putative long noncoding RNAs. Bioinformatics. 2015:2-3.

67. R Development Core Team. A language and environment for statistical computing. Vienna, Austria : the R foundation for statistical computing. 2011.

68. Robinson MD, McCarthy DJ, Smyth GK. edgeR: a bioconductor package for differential expression analysis of digital gene expression data. Bioinformatics. 2009;26:139-40.

69. Bohbot JD, Sparks JT, Dickens JC. The maxillary palp of Aedes Aegypti, a model of multisensory integration. Insect Biochem Mol Biol. 2014;48:29-39.

70. Bohbot J, Pitts RJ, Kwon H-W, Rützler M, Robertson HM, Zwiebel LJ. Molecular characterization of the Aedes Aegypti odorant receptor gene family. Insect Mol Biol. 2007;16:525-37.

71. Matthews BJ, McBride CS, DeGennaro M, Despo O, Vosshall LB. The neurotranscriptome of the Aedes Aegypti Mosquito. BMC Genomics. 2016;17:32.

72. Garneau NL, Sokoloski KJ, Opyrchal M, Neff CP, Wilusz CJ, Wilusz J. The 3' untranslated region of sindbis virus represses deadenylation of viral transcripts in mosquito and mammalian cells. J Virol. 2008:82:880-92.

73. Arca B, Lombardo F, Francischetti IM, Pham VM, Mestres-Simon M, Andersen JF, et al. An insight into the sialome of the adult female mosquito Aedes Albopictus. Insect Biochem Mol Biol. 2007;37:107-27.

74. Pitts R, Rinker D, Jones P. Transcriptome profiling of chemosensory appendages in the malaria vector Anopheles Gambiae reveals tissue-and sex-specific signatures of odor coding. BMC Genomics. 2011;12

75. Pelletier J, Leal WS. Genome analysis and expression patterns of odorantbinding proteins from the southern house mosquito Culex Pipiens Quinquefasciatus. PLoS One. 2009;4:e6237.

76. Pelletier J, Leal WS. Characterization of olfactory genes in the antennae of the southern house mosquito, Culex Quinquefasciatus. J Insect Physiol. 2011;57:915-29.

77. Liu C, Pitts RJ, Bohbot JD, Jones PL, Wang G, Zwiebel LJ. Distinct olfactory signaling mechanisms in the malaria vector mosquito Anopheles Gambiae. PLoS Biol. 2010;8

78. Yin J, Choo YM, Duan H, Leal WS. Selectivity of odorant-binding proteins from the southern house mosquito tested against physiologically relevant ligands. Front Physiol. 2015;6:1-8.

79. Sparks JT, Bohbot JD, Dickens JC. The genetics of chemoreception in the labella and tarsi of Aedes Aegypti. Insect Biochem Mol Biol. 2014;48:8-16.

80. Armbruster $P$, White S, Dzundza J, Crawford J, Zhao X. Identification of genes encoding atypical odorant-binding proteins in Aedes Albopictus (Diptera: Culicidae). J Med Entomol. 2009;46:271-80.

81. Li C, Yan T, Dong Y, Zhao T. Identification and quantitative analysis of genes encoding odorant binding proteins in Aedes Albopictus ( Diptera : Culicidae) identification and quantitative analysis of genes encoding odorant binding proteins in Aedes Albopictus ( Diptera : Culicidae ). Entomol Soc Am. 2012;49: 573-80.

82. Deng $\mathrm{Y}, \mathrm{Yan} \mathrm{H}, \mathrm{Gu} J, \mathrm{Xu} J, \mathrm{Wu} \mathrm{K}$, Tu Z, et al. Molecular and functional characterization of odorant-binding protein genes in an invasive vector mosquito, Aedes albopictus. PLoS One. 2013;8:e68836.

83. Pitts RJ, Rinker DC, Jones PL, Rokas A, Zwiebel LJ. Transcriptome profiling of chemosensory appendages in the malaria vector Anopheles Gambiae reveals tissue- and sex-specific signatures of odor coding. BMC Genomics. 2011;12:271.

84. Hoel DF, Kline DL, Allan SA, Grant A. Evaluation of carbon dioxide, 1-octen3-ol, and lactic acid as baits in mosquito magnettm pro traps for Aedes Albopictus in north Central Florida. J Am Mosq Control Assoc. 2007;23:11-7.

85. Neafsey D, Waterhouse R, Abai M. Highly evolvable malaria vectors: the genomes of 16 anopheles mosquitoes. Science. 2015:1-19.
86. Rinker DC, Zhou X, Pitts RJ, Rokas A, Zwiebel LJ. Antennal transcriptome profiles of anopheline mosquitoes reveal human host olfactory specialization in Anopheles Gambiae. BMC Genomics. 2013;14:749.

87. Scialò F, Hansson BS, Giordano E, Polito CL, Digilio FA. Molecular and functional characterization of the odorant receptor2 (OR2) in the tiger mosquito aedes albopictus. PLoS One. 2012;7:1-11.

88. Liu H, Liu T, Xie L, Wang X, Deng Y, Chen C-H, et al. Functional analysis of Orco and odorant receptors in odor recognition in Aedes Albopictus. Parasit Vectors. 2016;9:363.

89. Kim SM, Wang JW. Hygrosensation: feeling wet and cold. Curr Biol. 2016;26: R408-10.

90. Chen C, Buhl E, Xu M, Croset V, Rees JS, Lilley KS, et al. Drosophila Ionotropic receptor 25 a mediates circadian clock resetting by temperature. Nature. 2015;527:516-20

91. Kaupp UB. Olfactory signalling in vertebrates and insects: differences and commonalities. Nat Rev Neurosci. 2010;11:188-200.

92. Taparia $T$, Ignell $R$, Hill SR. Blood meal induced regulation of the chemosensory gene repertoire in the southern house mosquito. BMC Genomics. 2017;18:393.

93. Leal WS, Choo Y, Xu P, da Silva CSB, Ueira-Vieira C. Differential expression of olfactory genes in the southern house mosquito and insights into unique odorant receptor gene isoforms. Proc Natl Acad Sci U S A. 2013;1 10:18704-9.

94. Hodges TK. Cosme L V, Athrey G, Pathikonda S, Takken W, Slotman M a. Species-specific chemosensory gene expression in the olfactory organs of the malaria vector Anopheles Gambiae. BMC Genomics. 2014;15:1089-105.

95. Jaramillo Ramirez Gl, Logan JG, Loza-Reyes E, Stashenko E, Moores GD. Repellents inhibit P450 enzymes in Stegomyia (Aedes) aegypti. PLoS One. 2012;7:1-8.

96. Mao W, Schuler MA, Berenbaum MR. Task-related differential expression of four cytochrome P450 genes in honeybee appendages. Insect Mol Biol. 2015;24:582-8.

97. Ishida Y, Leal WS. Rapid inactivation of a moth pheromone. Proc Natl Acad Sci U S A. 2005:102:14075-9.

98. Vogt RG, Riddiford LM, Prestwich GD. Kinetic properties of a sex pheromone-degrading enzyme: the sensillar esterase of Antheraea Polyphemus. Proc Natl Acad Sci U S A. 1985;82:8827-31.

99. Sun W, Shen YH, Qi DW, Xiang ZH, Zhang Z. Molecular cloning and characterization of Ecdysone oxidase and 3-dehydroecdysone-3??-reductase involved in the ecdysone inactivation pathway of silkworm, Bombyx Mori. Int J Biol Sci. 2011;8:125-38.

100. Liu S, Gong Z-J, Rao X-J, Li M-Y, Li S-G. Identification of putative Carboxylesterase and glutathione $S$-transferase genes from the antennae of the Chilo suppressalis (Lepidoptera: Pyralidae). J Insect Sci. 2015;15:103.

101. Tan X, Hu XM, Zhong XW, Chen QM, Xia QY, Zhao P. Antenna-specific glutathione S-transferase in male silkmoth Bombyx Mori. Int J Mol Sci. 2014; 15:7429-43.

102. Balabanidou V, Kampouraki A, MacLean M, Blomquist GJ, Tittiger C, Juárez $M P$, et al. Cytochrome P450 associated with insecticide resistance catalyzes cuticular hydrocarbon production in Anopheles gambiae. Proc Natl Acad Sci. 2016;113:201608295

103. Enayati AA, Ranson H, Hemingway J. Insect glutathione transferases and insecticide resistance. Insect Mol Biol. 2005;14:3-8.

104. Justice RW, Dimitratos S, Walter MF, Woods DF, Biessmann H. Sexual dimorphic expression of putative antennal carrier protein genes in the malaria vector Anopheles Gambiae. Insect Mol Biol. 2003;12:581-94.

105. Zhou Y, Badgett MJ, Bowen JH, Vannini L, Orlando R, Willis JH. Distribution of cuticular proteins in different structures of adult Anopheles Gambiae. Insect Biochem Mol Biol. 2016:75:45-57.

106. Krieger J, Strobel J, Vogl A, Hanke W, Breer H. Identification of a cyclic nucleotide- and voltage-activated ion channel from insect antennae. Insect Biochem Mol Biol. 1999;29:255-67.

107. Freeman EG, Dahanukar A. Molecular neurobiology of drosophila taste. Curr Opin Neurobiol. 2015;34:140-8.

108. Gingl $E$, Hinterwirth A, Tichy $H$. Sensory representation of temperature in mosquito warm and cold cells. J Neurophysiol. 2005;94:176-85.

109. Shen WL, Kwon Y, Adegbola AA, Luo J, Chess A, Montell C. Function of Rhodopsin in temperature discrimination in drosophila. Science. 2011;331:1333-6.

110. Gurevich G. Arrestins: ubiquitous regulators of cellular signaling pathways. Genome Biol. 2006;7:236.

111. Alloway PG, Howard L, Dolph PJ. The formation of stable RhodopsinArrestin complexes induces apoptosis and photoreceptor cell degeneration. Neuron. 2000;28:129-38. 
112. Dolph PJ, Ranganathan R, Colley NJ, Hardy RW, Socolich M, Zuker CS. Arrestin function in inactivation of $\mathrm{G}$ protein-coupled receptor rhodopsin in vivo. Science. 1993;260:1910-6.

113. Merrill CE, Riesgo-Escovar J, Pitts RJ, Kafatos FC, Carlson JR, Zwiebel LJ. Visual arrestins in olfactory pathways of drosophila and the malaria vector mosquito Anopheles Gambiae. Proc Natl Acad Sci U S A. 2002;99:1633-8.

114. Zhang W, Yan Z, Jan LY, Jan YN. Sound response mediated by the TRP channels NOMPC, NANCHUNG, and INACTIVE in chordotonal organs of drosophila larvae. Proc Natl Acad Sci. 2013;110:13612-7.

115. Pennetier C, Warren B, Dabiré KR, Russell IJ, Gibson G. "Singing on the wing" as a mechanism for species recognition in the malarial mosquito Anopheles Gambiae. Curr Biol. 2010;20:131-6.

Submit your next manuscript to BioMed Central and we will help you at every step:

- We accept pre-submission inquiries

- Our selector tool helps you to find the most relevant journal

- We provide round the clock customer support

- Convenient online submission

- Thorough peer review

- Inclusion in PubMed and all major indexing services

- Maximum visibility for your research

Submit your manuscript at www.biomedcentral.com/submit
Biomed Central 